Research Article

\title{
Ranking Effectiveness of COVID-19 Tests Using Fuzzy Bipolar Soft Expert Sets
}

\author{
Ghous Ali, ${ }^{1}$ G. Muhiuddin $\left(\mathbb{D},{ }^{2}\right.$ Arooj Adeel, ${ }^{3}$ and Muhammad Zain Ul Abidin ${ }^{1}$ \\ ${ }^{1}$ Department of Mathematics, Division of Science \& Technology, University of Education, Lahore, Pakistan \\ ${ }^{2}$ Department of Mathematics, University of Tabuk, P. O. Box 741, Tabuk 71491, Saudi Arabia \\ ${ }^{3}$ Department of Mathematics, University of Education, Bank Road Campus, Lahore, Pakistan
}

Correspondence should be addressed to G. Muhiuddin; chishtygm@gmail.com

Received 23 April 2021; Accepted 13 July 2021; Published 31 July 2021

Academic Editor: Amin Jajarmi

Copyright $\odot 2021$ Ghous Ali et al. This is an open access article distributed under the Creative Commons Attribution License, which permits unrestricted use, distribution, and reproduction in any medium, provided the original work is properly cited.

The theory of fuzzy bipolar soft sets is an efficient extension of soft sets for depicting the bipolarity of uncertain fuzzy soft information; however, it is limited to a single expert. The present research article introduces the theory of an innovative hybrid model called the fuzzy bipolar soft expert sets, as a natural extension of two existing models (including fuzzy soft expert sets and fuzzy bipolar soft sets). The proposed model is highly suitable for describing the bipolarity of fuzzy soft information having multiple expert opinions. Some fundamental properties of the developed hybrid model are discussed, including subset, complement, union, intersection, AND operation, and OR operation. The proposed concepts are explained with detailed examples. Moreover, to demonstrate the applicability of our initiated model, an application of the proposed hybrid model is presented along with the developed algorithm to tackle the real-world group decision-making situation, that is, ranking effectiveness of tests in spread analysis of COVID-19. Finally, a comparative analysis of the developed model with some existing mathematical tools such as fuzzy soft expert sets and fuzzy bipolar soft sets is provided to show the cogency and reliability of the initiated model.

\section{Introduction}

The ranking and selection of alternatives (based on the preferences of decision-makers) is an important aspect of decision sciences, but the situations become difficult when dealing with vague data and uncertainties. The conception of the modern theory of probability in the sixteenth century led to the proposal of different mathematical tools and algorithms by many computer, logics, and mathematical experts in order to deal with uncertainty and fuzziness, whether considering social sciences or economics, medical sciences, or engineering. In 1965, Zadeh [1] initiated the idea of a fuzzy set model which is capable of handling partial truth between "absolute false" and "absolute truth." In such concepts of fuzzy sets, it is declared that an element of a universal set $\mathcal{U}$ can have some membership degree belonging to interval $[0,1]$ instead of the set $\{0,1\}$, thus allowing to deal with situations considering "how much" an element satisfies a criteria instead of just declaring whether it satisfies or not. This powerful concept of fuzziness was later used by various scientific researchers from almost every scientific domain. One important limitation of this model is to deal with bipolar or dual behaviour situations involving a positive and a negative side, for instance, effect and side effect, good and bad, pros and cons. To remove this limitation, Zhang [2] presented Yin-Yang bipolar fuzzy sets which are capable of handling these bipolar situations. Yin and Yang are considered as negative and positive parts, respectively, of a system in Chinese medicine.

The fuzzy set theory led to the development and proposal of extensions like intuitionistic fuzzy sets [3] and bipolar fuzzy sets [2]. However, these models fail to deal with uncertain situations involving different parameters. This limitation due to lack of parameterization tools in existing models led Molodtsov [4] to initiate the notion of soft sets, which is different from all other preexisting methods and allows to deal with situations having different parameters. The soft set theory has been used in several domains 
including medicine, engineering, and economics. Certain applications of such a model in decision-making were offered by Maji et al. [5]. Furthermore, Ali et al. [6] studied several properties of the soft sets. Later on, such a model was combined with existing uncertain models to develop the powerful hybrid models incorporating the characteristics of both the combined models such as fuzzy soft sets [7].

The soft set model is a powerful tool, but fails to deal with two-sided information in a soft environment having interrelated parameters, where one parameter affects the other one, such as "expensive and cheap," "old and young," and "dependent and independent." To tackle this situation, the idea of bipolar soft sets (BSSs) was proposed by Shabir and $\mathrm{Naz}$ [8]. The BSS model considers two opposite meaning sets of parameters named the "set of parameters" and the "not set of parameters." Thus, dealing with bipolar parameters much efficiently, Naz and Shabir [9] combined fuzzy set theory with BSSs to introduce the fuzzy BSSs and further discussed their decision-making applications. Akram and Ali [10] introduced Pythagorean fuzzy BSSs and rough Pythagorean fuzzy BSSs and discussed their applications. Afterwards, Akram et al. [11] introduced two new decision-making models, including $m$-polar fuzzy BSSs and rough $m$-polar fuzzy BSSs. A survey on hybrid soft set models was launched by Ma et al. [12]. Later, Mahmood [13] presented a novel decision-making method for BSSs and studied some of their practical applications.

Hybrid models proved to be an important tool in dealing with group decision-making problems. Many researchers across the globe constructed novel hybrid models that give more reliable outputs, when dealing with different sorts of information pieces in decision-making problems. In the last few decades, hybrid models have been developed to tackle numerous real-world multiattribute group decision-making (MAGDM) situations containing vague information. All the abovementioned models deal with only one expert and fail to consider the opinion of multiple experts in the same place and are not considered suitable in MAGDM situations having multiple expert opinions as in the case of studies utilizing questionnaires. Alkazellah and Salleh [14] resolved this issue by introducing the notion of soft expert sets (SESs), which considers all expert opinions dealing with a MAGDM situation. Later on, Alkazellah and Salleh [15] presented fuzzy soft expert sets (FSESs). This powerful concept inspired many researchers to solve different MAGDM problems using the SES approach, as discussed in [16-21]. For instance, Broumi and Smarandache [22] presented an intuitionistic fuzzy SES model and discussed its applications. Hassan and Alhazaymeh [23] introduced vague SESs. Shabir and Gul [24] developed modified rough BSSs. Recently, Ali and Akram [25] introduced $\mathrm{N}$-SESs and fuzzy $\mathrm{N}$-SESs with their use in MAGDM situations. In addition, Akram et al. [26] launched a novel hybrid model called $m$-polar fuzzy SESs and solved certain MAGDM problems. For more important terminologies, the readers are referred to [27-39].

The motivations of this research article are described as follows:
(1) The ability of BSSs and fuzzy BSSs is depicting bipolar information in a soft environment, which allows to deal with bipolar parameters more effectively, but not so efficient in group decisions.

(2) SESs and fuzzy SESs appear as powerful tools in MAGDM problems allowing multiple expert opinions in the same model. Much effective model is possible, if it deals with bipolarities.

The major contributions of this study are described as follows:

(1) The bipolarity of fuzzy BSSs is merged with the group decision-making skills of fuzzy SESs to develop a more powerful, more effective, new model called fuzzy bipolar SESs or FBSESs, which is capable of dealing with situations involving multiple experts as well as bipolar parameters in a fuzzy environment.

(2) The dominant properties as well as the notable results about the proposed model are explained, including subset, complement, union, intersection, AND operation, and OR operation. In addition, supportive numerical examples are provided.

(3) A real-world MAGDM problem to evaluate the ranking effectiveness of tests for spread analysis of COVID-19 has been solved with the proposed model.

(4) An effective algorithm to solve MAGDM problems with FBSESs is provided.

(5) At the end, advantages, comparative analysis, and limitations of the proposed model are discussed, to prove the effectiveness and novelty of the developed FBSES model.

The research article is organized as follows. In Section 2 , we present a new hybrid mathematical tool called FBSESs and discuss some of its fundamental properties including subset, complement, agree FBSES, disagree FBSES, AND operation, and OR operation. In Section 3, we discuss a real-world MAGDM problem concerning the emerging COVID-19 using the FBSES approach. We also provide an efficient algorithm for the developed model in this section. Section 4 provides a comparative analysis of the developed method with certain preexisting models, including fuzzy BSSs and fuzzy SESs. Finally, in Section 5, we provide some concluding remarks and future orientations.

\section{Fuzzy Bipolar Soft Expert Sets}

This section reviews some basic terminologies and provides the notion of FBSESs with necessary properties of these concepts along with detailed supporting examples.

Definition 1 (see [8]). Let $\mathcal{U}$ be a universe and $\mathscr{V}$ be the universe of parameters. For every $\mathscr{B} \subseteq \mathscr{V}$, a triplet $(F, G, \mathscr{B})$ is said to be a bipolar soft set or BSS on $\mathcal{U}$, where $F$ and $G$ are functions defined as follows: 


$$
\begin{aligned}
& F: \mathscr{B} \longrightarrow \mathscr{P}(\mathcal{U}), \\
& \text { G: } \mathscr{B} \longrightarrow \mathscr{P}(\mathcal{U}),
\end{aligned}
$$

such that $F(\vartheta) \cap G(\vartheta)=\varnothing, \forall \vartheta \in \mathscr{B}, \vartheta \in \mathscr{B}$. Here, $\mathscr{P}(\mathcal{U})$ represents the power set of $\mathscr{U}$.

Notice that $\mathscr{B}($ Not $\mathscr{B})$ is the set containing attributes opposite to those contained in set $\mathscr{B}$.

Definition 2 (see [15]). Let $\mathcal{U}$ be the universe, $\mathscr{V}$ the universe of parameters, and $E$ the set of experts. Let $X=\mathscr{V} \times E \times \mathcal{O}$, where $\mathcal{O}=\{0=$ disagree, $1=$ agree $\}$ represents the set of opinions. Then, for $\mathscr{A} \subseteq X$, a pair $(\gamma, \mathscr{A})$ is said to be a fuzzy soft expert set over the universe $\mathcal{U}$, where $\gamma$ is the function defined as follows:

$$
\gamma: \mathscr{A} \longrightarrow \mathscr{F}(\mathcal{U}),
$$

where $\mathscr{F}(\mathcal{U})$ serves as the power set of fuzzy subsets over $\mathcal{U}$.

Now, we are ready to define the fuzzy bipolar soft expert sets.

Definition 3. Let $\mathcal{U}$ be the universal set and $\mathscr{V}$ be the universe of parameters. Let $E$ be the set of experts, $\mathcal{O}=$ $\{0=$ disagree, $1=$ agree $\}$ the set of opinions, and $X=\mathscr{V} \times E \times \mathcal{O}$. Then, the triplet $(\gamma, \chi, \mathscr{A})$ is called a fuzzy bipolar soft expert set or FBSES on $\mathcal{U}$, where $\gamma$ and $\chi$ are functions defined as follows:

$$
\begin{aligned}
& \gamma: \mathscr{A} \longrightarrow \mathscr{F}(\mathcal{U}), \\
& \chi: \mathscr{A} \longrightarrow \mathscr{F}(\mathcal{U}),
\end{aligned}
$$

with $\mathscr{A} \subseteq X$ and $\mathscr{A} \subseteq X$, such that $0 \leq(\gamma(\alpha))(u)+(\chi(\alpha))$ $(u) \leq 1$ for all $\alpha \in \mathscr{A}$ and $u \in \mathcal{U}$.

Note 1. $\mathscr{A}($ not $\mathscr{A})$ is the set containing attributes opposite to those contained in $\mathscr{A}$.

Here, $0 \leq(\gamma(\alpha))(u)+(\chi(\alpha))(u) \leq 1$ for all $\alpha \in \mathscr{A}$ and $u \in \mathcal{U}$ acts as a condition for $\gamma$ and $\chi$ to be consistent with the definition of FBSESs. The functions $\gamma(\alpha)$ and $\chi(\alpha)$ are considered as the $\alpha$-approximate and $\alpha$-approximate elements of the FBSES $(\gamma, \chi, \mathscr{A})$ for each $\alpha \in \mathscr{A}$.

Definition 4. Let $(\gamma, \chi, \mathscr{A})$ be a FBSES over a universe $\mathcal{U}$, then the hesitancy region of this FBSES is determined by a fuzzy soft expert set $(\mathfrak{H}, \mathscr{A})$, such that

$$
(\mathfrak{H}(\alpha))(u)=1-\{(\gamma(\alpha))(u)+(\chi(\alpha))(u)\},
$$

for all $\alpha \in \mathscr{A}$ and each $u \in \mathscr{U}$.

Here, $(\mathfrak{H}, \mathscr{A})$ represents the grey area or the uncertainty in making the decision, thus indicating the lack of knowledge of the expert in the case of a particular object with respect to a parameter under consideration. Moreover, $\gamma(\alpha))(u)+\chi(\alpha))(u)+\mathfrak{H}(\alpha))(u)=1$ for all $\alpha \in \mathscr{A}, \alpha \in \mathscr{A}$, and for all $u \in \mathcal{U}$.

Example 1. Consider that 5 patients are monitored in a mental health facility for the diagnosis of their mental state by a group of 3 psychiatrists. Let $\mathcal{U}=\left\{u_{1}, u_{2}, \ldots, u_{5}\right\}$ be the set of patients. Let $\mathscr{V}=\left\{\vartheta_{1}, \vartheta_{2}, \vartheta_{3}\right\}$
$=\{$ very quiet, tearfulness or crying diffidence $\}$ be the set of manic behaviours, whereas $\mathscr{V}=\left\{\vartheta_{1}, \vartheta_{2}, \vartheta_{3}\right\}$ $=\{$ very quiet, tearfulness or crying diffidence $\}$ be the set of respective depressive behaviours. Let $E=\{x, y, z\}$ be the set of psychiatrists.

After monitoring the patients for a fixed period of time, the psychiatrists make data on the basis of their observations and we get the FBSES $(\gamma, \chi, X)$ as follows:

$$
\begin{aligned}
& (\gamma, X)=\left\{\left(\left(\vartheta_{1}, x, 1\right),\left\{\left(u_{1}, 0.1\right),\left(u_{2}, 0.3\right),\left(u_{3}, 0.7\right),\left(u_{4}, 0.2\right),\left(u_{5}, 0.5\right)\right\}\right),\right. \\
& \left(\left(\vartheta_{1}, y, 1\right),\left\{\left(u_{1}, 0.1\right),\left(u_{2}, 0.4\right),\left(u_{3}, 0.8\right),\left(u_{4}, 0.1\right),\left(u_{5}, 0.4\right)\right\}\right), \\
& \left(\left(\vartheta_{1}, z, 1\right),\left\{\left(u_{1}, 0.2\right),\left(u_{2}, 0.25\right),\left(u_{3}, 0.7\right),\left(u_{4}, 0.2\right),\left(u_{5}, 0.4\right)\right\}\right), \\
& \left(\left(\vartheta_{2}, x, 1\right),\left\{\left(u_{1}, 0\right),\left(u_{2}, 0.4\right),\left(u_{3}, 0.8\right),\left(u_{4}, 0.1\right),\left(u_{5}, 0.3\right)\right\}\right), \\
& \left(\left(\vartheta_{2}, y, 1\right),\left\{\left(u_{1}, 0.05\right),\left(u_{2}, 0.5\right),\left(u_{3}, 0.7\right),\left(u_{4}, 0.1\right),\left(u_{5}, 0.35\right)\right\}\right), \\
& \left(\left(\vartheta_{2}, z, 1\right),\left\{\left(u_{1}, 0\right),\left(u_{2}, 0.2\right),\left(u_{3}, 0.6\right),\left(u_{4}, 0.15\right),\left(u_{5}, 0.4\right)\right\}\right), \\
& \left(\left(\vartheta_{3}, x, 1\right),\left\{\left(u_{1}, 0\right),\left(u_{2}, 0.3\right),\left(u_{3}, 0.7\right),\left(u_{4}, 0.2\right),\left(u_{5}, 0.3\right)\right\}\right), \\
& \left(\left(\vartheta_{3}, y, 1\right),\left\{\left(u_{1}, 0.05\right),\left(u_{2}, 0.4\right),\left(u_{3}, 0.5\right),\left(u_{4}, 0.1\right),\left(u_{5}, 0.4\right)\right\}\right), \\
& \left(\left(\vartheta_{3}, z, 1\right),\left\{\left(u_{1}, 0\right),\left(u_{2}, 0.3\right),\left(u_{3}, 0.7\right),\left(u_{4}, 0.2\right),\left(u_{5}, 0.5\right)\right\}\right), \\
& \left(\left(\vartheta_{1}, x, 0\right),\left\{\left(u_{1}, 0.8\right),\left(u_{2}, 0.5\right),\left(u_{3}, 0.1\right),\left(u_{4}, 0.5\right),\left(u_{5}, 0.5\right)\right\}\right), \\
& \left(\left(\vartheta_{1}, y, 0\right),\left\{\left(u_{1}, 0.9\right),\left(u_{2}, 0.5\right),\left(u_{3}, 0.2\right),\left(u_{4}, 0.5\right),\left(u_{5}, 0.5\right)\right\}\right), \\
& \left(\left(\vartheta_{1}, z, 0\right),\left\{\left(u_{1}, 0.8\right),\left(u_{2}, 0.6\right),\left(u_{3}, 0.2\right),\left(u_{4}, 0.5\right),\left(u_{5}, 0.4\right)\right\}\right), \\
& \left(\left(\vartheta_{2}, x, 0\right),\left\{\left(u_{1}, 1\right),\left(u_{2}, 0.5\right),\left(u_{3}, 0.1\right),\left(u_{4}, 0.4\right),\left(u_{5}, 0.5\right)\right\}\right), \\
& \left(\left(\vartheta_{2}, y, 0\right),\left\{\left(u_{1}, 0.9\right),\left(u_{2}, 0.4\right),\left(u_{3}, 0.2\right),\left(u_{4}, 0.5\right),\left(u_{5}, 0.6\right)\right\}\right), \\
& \left(\left(\vartheta_{2}, z, 0\right),\left\{\left(u_{1}, 0.9\right),\left(u_{2}, 0.8\right),\left(u_{3}, 0.3\right),\left(u_{4}, 0.5\right),\left(u_{5}, 0.5\right)\right\}\right), \\
& \left(\left(9_{3}, x, 0\right),\left\{\left(u_{1}, 0.8\right),\left(u_{2}, 0.6\right),\left(u_{3}, 0.1\right),\left(u_{4}, 0.4\right),\left(u_{5}, 0.6\right)\right\}\right), \\
& \left(\left(\vartheta_{3}, y, 0\right),\left\{\left(u_{1}, 0.8\right),\left(u_{2}, 0.5\right),\left(u_{3}, 0.3\right),\left(u_{4}, 0.5\right),\left(u_{5}, 0.5\right)\right\}\right), \\
& \left.\left(\left(\vartheta_{3}, z, 0\right),\left\{\left(u_{1}, 0.9\right),\left(u_{2}, 0.5\right),\left(u_{3}, 0.2\right),\left(u_{4}, 0.5\right),\left(u_{5}, 0.4\right)\right\}\right)\right\}, \\
& (\chi, X)=\left\{\left(\left(\vartheta_{1}, x, 1\right),\left\{\left(u_{1}, 0.8\right),\left(u_{2}, 0.4\right),\left(u_{3}, 0\right),\left(u_{4}, 0.2\right),\left(u_{5}, 0.4\right)\right\}\right),\right. \\
& \left(\left(\vartheta_{1}, y, 1\right),\left\{\left(u_{1}, 0.85\right),\left(u_{2}, 0.35\right),\left(u_{3}, 0.1\right),\left(u_{4}, 0.1\right),\left(u_{5}, 0.5\right)\right\}\right), \\
& \left(\left(\vartheta_{1}, z, 1\right),\left\{\left(u_{1}, 0.7\right),\left(u_{2}, 0.25\right),\left(u_{3}, 0.2\right),\left(u_{4}, 0.2\right),\left(u_{5}, 0.4\right)\right\}\right), \\
& \left(\left(\vartheta_{2}, x, 1\right),\left\{\left(u_{1}, 0.6\right),\left(u_{2}, 0.3\right),\left(u_{3}, 0\right),\left(u_{4}, 0\right),\left(u_{5}, 0.35\right)\right\}\right), \\
& \left(\left(\vartheta_{2}, y, 1\right),\left\{\left(u_{1}, 0.7\right),\left(u_{2}, 0.3\right),\left(u_{3}, 0\right),\left(u_{4}, 0.05\right),\left(u_{5}, 0.4\right)\right\}\right), \\
& \left(\left(\vartheta_{2}, z, 1\right),\left\{\left(u_{1}, 0.6\right),\left(u_{2}, 0.25\right),\left(u_{3}, 0\right),\left(u_{4}, 0.1\right),\left(u_{5}, 0.4\right)\right\}\right) \text {, } \\
& \left(\left(\vartheta_{3}, x, 1\right),\left\{\left(u_{1}, 0.8\right),\left(u_{2}, 0.3\right),\left(u_{3}, 0.1\right),\left(u_{4}, 0.1\right),\left(u_{5}, 0.5\right)\right\}\right), \\
& \left(\left(\vartheta_{3}, y, 1\right),\left\{\left(u_{1}, 0.8\right),\left(u_{2}, 0.3\right),\left(u_{3}, 0.1\right),\left(u_{4}, 0.15\right),\left(u_{5}, 0.4\right)\right\}\right), \\
& \left(\left(\vartheta_{3}, z, 1\right),\left\{\left(u_{1}, 0.9\right),\left(u_{2}, 0.4\right),\left(u_{3}, 0.05\right),\left(u_{4}, 0.1\right),\left(u_{5}, 0.4\right)\right\}\right), \\
& \left(\left(\vartheta_{1}, x, 0\right),\left\{\left(u_{1}, 0.1\right),\left(u_{2}, 0.5\right),\left(u_{3}, 0.8\right),\left(u_{4}, 0.5\right),\left(u_{5}, 0.4\right)\right\}\right), \\
& \left(\left(\vartheta_{1}, y, 0\right),\left\{\left(u_{1}, 0.1\right),\left(u_{2}, 0.4\right),\left(u_{3}, 0.8\right),\left(u_{4}, 0.5\right),\left(u_{5}, 0.4\right)\right\}\right), \\
& \left(\left(\vartheta_{1}, z, 0\right),\left\{\left(u_{1}, 0.2\right),\left(u_{2}, 0.4\right),\left(u_{3}, 0.7\right),\left(u_{4}, 0.5\right),\left(u_{5}, 0.4\right)\right\}\right), \\
& \left(\left(\vartheta_{2}, x, 0\right),\left\{\left(u_{1}, 0\right),\left(u_{2}, 0.5\right),\left(u_{3}, 0.8\right),\left(u_{4}, 0.5\right),\left(u_{5}, 0.5\right)\right\}\right) \text {, } \\
& \left(\left(\vartheta_{2}, y, 0\right),\left\{\left(u_{1}, 0.05\right),\left(u_{2}, 0.5\right),\left(u_{3}, 0.7\right),\left(u_{4}, 0.4\right),\left(u_{5}, 0.35\right)\right\}\right), \\
& \left(\left(\vartheta_{2}, z, 0\right),\left\{\left(u_{1}, 0.1\right),\left(u_{2}, 0.2\right),\left(u_{3}, 0.6\right),\left(u_{4}, 0.5\right),\left(u_{5}, 0.4\right)\right\}\right), \\
& \left(\left(\vartheta_{3}, x, 0\right),\left\{\left(u_{1}, 0.1\right),\left(u_{2}, 0.4\right),\left(u_{3}, 0.7\right),\left(u_{4}, 0.6\right),\left(u_{5}, 0.3\right)\right\}\right), \\
& \left(\left(\vartheta_{3}, y, 0\right),\left\{\left(u_{1}, 0.1\right),\left(u_{2}, 0.4\right),\left(u_{3}, 0.5\right),\left(u_{4}, 0.5\right),\left(u_{5}, 0.4\right)\right\}\right), \\
& \left.\left(\left(\vartheta_{3}, z, 0\right),\left\{\left(u_{1}, 0\right),\left(u_{2}, 0.4\right),\left(u_{3}, 0.7\right),\left(u_{4}, 0.4\right),\left(u_{5}, 0.5\right)\right\}\right)\right\} \text {. }
\end{aligned}
$$

This FBSES shows the observations of psychiatrists during the diagnosis. For example, psychiatrist $x$ declares " $u_{1}$ " to be $10 \%$ talkative, $80 \%$ quiet, and $10 \%$ normal (grey 
area) during the diagnosis, whereas $y$ declares " $u_{1}$ " to be $10 \%$ talkative, $85 \%$ quiet, while acting normal $5 \%$ of the time. On the basis of the collected data, patients having high membership degrees in manic behaviours are declared as hypomania patients, those with high membership degrees in depressive behaviours are declared to be under depression, whereas the patients with almost equal degrees of membership in both manic and depressive conditions (above $30 \%-35 \%$ ) are declared to be suffering with bipolar disorder. Thus, the above set shows that the psychiatrist $x$ considers " $u_{1}$ " to be under depression, " $u_{3}$ " to be suffering with hypomania, " $u_{2}$ and $u_{5}$ " to be bipolar, while " $u_{4}$ " to behave like a normal person, and so on.

Tables 1 and 2 represent the FBSES in Example 1 with respect to $\gamma$ and $\chi$ functions, respectively, whereas Table 3 gives a single table representation of the above FBSES, such that entries $a_{i j}$ of the table of $i$-th rows and $j$-th columns are represented as

$$
a_{i j}=\left(\gamma_{i j}, \chi_{i j}\right)
$$

Definition 5. For two FBSESs $(\gamma, \chi, \mathscr{A})$ and $\left(\gamma_{1}, \chi_{1}, \mathscr{B}\right)$ on the universe $\mathscr{U},(\gamma, \chi, \mathscr{A})$ is called a fuzzy bipolar soft expert subset of $\left(\gamma_{1}, \chi_{1}, \mathscr{B}\right)$ if

\section{(1) $\mathscr{A} \subseteq \mathscr{B}$}

(2) $\gamma(\alpha) \subseteq \gamma_{1}(\alpha)$ and $\chi_{1}(\alpha) \subseteq \chi(\alpha)$ for all $\alpha \in \mathscr{A}$. In other words, $\quad(\gamma(\alpha))(u) \leq\left(\gamma_{1}(\alpha)\right)(u)$ and $\left(\chi_{1}(\alpha)\right)(u)$ $\leq(\chi(\alpha))(u)$ for all values of $\alpha \in \mathscr{A}$ and $u \in \mathcal{U}$.

It is denoted by $(\gamma, \chi, \mathscr{A}) \hat{\subseteq}\left(\gamma_{1}, \chi_{1}, \mathscr{B}\right)$. Similarly, $\left(\gamma_{1}, \chi_{1}, \mathscr{B}\right)$ is a fuzzy bipolar soft expert superset of $(\gamma, \chi, \mathscr{A})$, and the superset relation is then denoted as $\left(\gamma_{1}, \chi_{1}, \mathscr{B}\right) \hat{\supseteq}(\gamma, \chi, \mathscr{A})$.

Definition 6. Any two FBSESs $(\gamma, \chi, \mathscr{A})$ and $\left(\gamma_{1}, \chi_{1}, \mathscr{B}\right)$ are said to be equal over a universe $\mathcal{U}$ if and only if $(\gamma, \chi, \mathscr{A})$ is a subset of $\left(\gamma_{1}, \chi_{1}, \mathscr{B}\right)$ and $\left(\gamma_{1}, \chi_{1}, \mathscr{B}\right)$ is a subset of $(\gamma, \chi, \mathscr{A})$.

Example 2. Consider Example 1. After treating the patients for a month, the psychiatrists again diagnose the patients on the basis of their behaviours.

Let $\mathscr{A}=\left\{\left(\vartheta_{1}, x, 1\right),\left(\vartheta_{2}, x, 0\right),\left(\vartheta_{3}, y, 1\right)\right\} \quad$ and $\mathscr{B}=\left\{\left(\vartheta_{1}, x, 1\right),\left(\vartheta_{1}, z, 1\right),\left(\vartheta_{2}, x, 0\right),\left(\vartheta_{3}, y, 1\right)\right\}$ be the subsets of $X=\mathscr{V} \times E \times \mathscr{O}$. Suppose two FBSESs $(\gamma, \chi, \mathscr{A})$ and $\left(\gamma_{1}, \chi_{1}, \mathscr{B}\right)$ are defined in Tables 4 and 5 , respectively.

Here, we see that $\mathscr{A} \subset \mathscr{B}$. In addition, $\gamma_{1}(\alpha) \supseteq \gamma(\alpha)$ and $\chi_{1}(\alpha) \subseteq \chi(\alpha)$ for all $\alpha \in \mathscr{A}$. Clearly, $(\gamma, \chi, \mathscr{A}) \widehat{\subseteq}\left(\gamma_{1}, \chi_{1}, \mathscr{B}\right)$.

Definition 7. The complement of a FBSES $(\gamma, \chi, \mathscr{A})$ over universe $\mathcal{U}$ is denoted by $(\gamma, \chi, \mathscr{A})^{c}$ and is defined by $(\gamma, \chi, \mathscr{A})^{c}=\left(\gamma^{c}, \chi^{c}, \mathscr{A}\right)$, where $\gamma^{c}$ and $\chi^{c}$ are functions given as $\gamma^{c}(\alpha)=\chi(\alpha)$ and $\chi^{c}(\alpha)=\gamma(\alpha)$ for all $\alpha \in \mathscr{A}, \alpha \in \mathscr{A}$.

Proposition 1. If $(\gamma, \chi, \mathscr{A})$ is a FBSES on $\mathcal{U}$, then

(1) $\left((\gamma, \chi, \mathscr{A})^{c}\right)^{c}=(\gamma, \chi, \mathscr{A})$
TABLe 1: $(\gamma, X)$

\begin{tabular}{lccccc}
\hline$(\gamma, X)$ & $u_{1}$ & $u_{2}$ & $u_{3}$ & $u_{4}$ & $u_{5}$ \\
\hline$\left(\vartheta_{1}, x, 1\right)$ & 0.1 & 0.3 & 0.7 & 0.2 & 0.5 \\
$\left(\vartheta_{1}, y, 1\right)$ & 0.1 & 0.4 & 0.8 & 0.1 & 0.4 \\
$\left(\vartheta_{1}, z, 1\right)$ & 0.2 & 0.25 & 0.7 & 0.2 & 0.4 \\
$\left(\vartheta_{2}, x, 1\right)$ & 0.0 & 0.4 & 0.8 & 0.1 & 0.3 \\
$\left(\vartheta_{2}, y, 1\right)$ & 0.05 & 0.5 & 0.7 & 0.1 & 0.35 \\
$\left(\vartheta_{2}, z, 1\right)$ & 0.0 & 0.2 & 0.6 & 0.15 & 0.4 \\
$\left(\vartheta_{3}, x, 1\right)$ & 0.0 & 0.3 & 0.7 & 0.2 & 0.3 \\
$\left(\vartheta_{3}, y, 1\right)$ & 0.05 & 0.4 & 0.5 & 0.1 & 0.4 \\
$\left(\vartheta_{3}, z, 1\right)$ & 0.0 & 0.3 & 0.7 & 0.2 & 0.5 \\
$\left(\vartheta_{1}, x, 0\right)$ & 0.8 & 0.5 & 0.1 & 0.5 & 0.5 \\
$\left(\vartheta_{1}, y, 0\right)$ & 0.9 & 0.5 & 0.2 & 0.5 & 0.5 \\
$\left(\vartheta_{1}, z, 0\right)$ & 0.8 & 0.6 & 0.2 & 0.5 & 0.4 \\
$\left(\vartheta_{2}, x, 0\right)$ & 1.0 & 0.5 & 0.1 & 0.4 & 0.5 \\
$\left(\vartheta_{2}, y, 0\right)$ & 0.9 & 0.4 & 0.2 & 0.5 & 0.6 \\
$\left(\vartheta_{2}, z, 0\right)$ & 0.9 & 0.8 & 0.3 & 0.5 & 0.5 \\
$\left(\vartheta_{3}, x, 0\right)$ & 0.8 & 0.6 & 0.1 & 0.4 & 0.6 \\
$\left(\vartheta_{3}, y, 0\right)$ & 0.8 & 0.5 & 0.3 & 0.5 & 0.5 \\
$\left(\vartheta_{3}, z, 0\right)$ & 0.9 & 0.5 & 0.2 & 0.5 & 0.4 \\
\hline
\end{tabular}

TABLE 2: $(\chi, X)$

\begin{tabular}{lccccc}
\hline$(\chi, X)$ & $u_{1}$ & $u_{2}$ & $u_{3}$ & $u_{4}$ & $u_{5}$ \\
\hline$\left(\vartheta_{1}, x, 1\right)$ & 0.8 & 0.4 & 0.0 & 0.2 & 0.4 \\
$\left(\vartheta_{1}, y, 1\right)$ & 0.85 & 0.35 & 0.1 & 0.1 & 0.5 \\
$\left(\vartheta_{1}, z, 1\right)$ & 0.7 & 0.25 & 0.2 & 0.2 & 0.4 \\
$\left(\vartheta_{2}, x, 1\right)$ & 0.6 & 0.3 & 0.0 & 0.0 & 0.35 \\
$\left(\vartheta_{2}, y, 1\right)$ & 0.7 & 0.3 & 0.0 & 0.05 & 0.4 \\
$\left(\vartheta_{2}, z, 1\right)$ & 0.6 & 0.25 & 0.0 & 0.1 & 0.4 \\
$\left(\vartheta_{3}, x, 1\right)$ & 0.8 & 0.3 & 0.1 & 0.1 & 0.5 \\
$\left(\vartheta_{3}, y, 1\right)$ & 0.8 & 0.3 & 0.1 & 0.15 & 0.4 \\
$\left(\vartheta_{3}, z, 1\right)$ & 0.9 & 0.4 & 0.05 & 0.1 & 0.4 \\
$\left(\vartheta_{1}, x, 0\right)$ & 0.1 & 0.5 & 0.8 & 0.5 & 0.4 \\
$\left(\vartheta_{1}, y, 0\right)$ & 0.1 & 0.4 & 0.8 & 0.5 & 0.4 \\
$\left(\vartheta_{1}, z, 0\right)$ & 0.2 & 0.4 & 0.7 & 0.5 & 0.4 \\
$\left(\vartheta_{2}, x, 0\right)$ & 0.0 & 0.5 & 0.8 & 0.5 & 0.5 \\
$\left(\vartheta_{2}, y, 0\right)$ & 0.05 & 0.5 & 0.7 & 0.4 & 0.35 \\
$\left(\vartheta_{2}, z, 0\right)$ & 0.1 & 0.2 & 0.6 & 0.5 & 0.4 \\
$\left(\vartheta_{3}, x, 0\right)$ & 0.1 & 0.4 & 0.7 & 0.6 & 0.3 \\
$\left(\vartheta_{3}, y, 0\right)$ & 0.1 & 0.4 & 0.5 & 0.5 & 0.4 \\
$\left(\vartheta_{3}, z, 0\right)$ & 0.0 & 0.4 & 0.7 & 0.4 & 0.5 \\
\hline & & & & &
\end{tabular}

Proof

(1) From Definition 7, we have $(\gamma, \chi, \mathscr{A})^{c}=\left(\gamma^{c}, \chi^{c}, \mathscr{A}\right)$ such that $\gamma^{c}(\alpha)=\chi(\alpha)$ and $\chi^{c}(\alpha)=\gamma(\alpha)$. Now

$$
\begin{aligned}
& \left((\gamma(\alpha))^{c}\right)^{c}=(\chi(\alpha))^{c}=\gamma(\alpha), \\
& \left((\chi(\alpha))^{c}\right)^{c}=(\gamma(\alpha))^{c}=\chi(\alpha) .
\end{aligned}
$$

Hence, $\left((\gamma, \chi, \mathscr{A})^{c}\right)^{c}=(\gamma, \chi, \mathscr{A})$.

Example 3. Consider the FBSES $(\gamma, \chi, \mathscr{A})$ in Example 2. Its complement $(\gamma, \chi, \mathscr{A})^{c}$ is defined in Table 6 .

Definition 8. A FBSES over the universe $\mathcal{U}$ is called a relative null FBSES, denoted by $(\Phi, \mathcal{U}, \mathscr{A})$, if $\Phi(\alpha)=\varnothing$ (i.e., $(\Phi(\alpha))(u)=0, \forall \alpha \in \mathscr{A}$ and $u \in \mathscr{U}$ ) and $\mathcal{U}(\alpha)=\mathscr{U}$ (i.e., $(\mathfrak{U}(\alpha))(u)=1, \forall \alpha \in \mathscr{A}$ and $u \in \mathscr{U})$, for all $\alpha \in \mathscr{A}, \alpha \in \mathscr{A}$. 
TABLe 3: Tabular form of FBSES $(\gamma, \chi, X)$.

\begin{tabular}{|c|c|c|c|c|c|}
\hline$(\gamma, \chi, X)$ & $u_{1}$ & $u_{2}$ & $u_{3}$ & $u_{4}$ & $u_{5}$ \\
\hline$\left(\vartheta_{1}, x, 1\right)$ & $(0.1,0.8)$ & $(0.3,0.4)$ & $(0.7,0.0)$ & $(0.2,0.2)$ & $(0.5,0.4)$ \\
\hline$\left(\vartheta_{1}, y, 1\right)$ & $(0.1, .85)$ & $(0.4, .35)$ & $(0.8,0.1)$ & $(0.1,0.1)$ & $(0.4,0.5)$ \\
\hline$\left(\vartheta_{1}, z, 1\right)$ & $(0.2,0.7)$ & $(.25, .25)$ & $(0.7,0.2)$ & $(0.2,0.2)$ & $(0.4,0.4)$ \\
\hline$\left(\vartheta_{2}, x, 1\right)$ & $(0.0,0.6)$ & $(0.4,0.3)$ & $(0.8,0.0)$ & $(0.1,0.0)$ & $(0.3, .35)$ \\
\hline$\left(\vartheta_{2}, y, 1\right)$ & $(.05,0.7)$ & $(0.5,0.3)$ & $(0.7,0.0)$ & $(0.1, .05)$ & $(.35,0.4)$ \\
\hline$\left(\vartheta_{2}, z, 1\right)$ & $(0.0,0.6)$ & $(0.2, .25)$ & $(0.6,0.0)$ & $(.15,0.1)$ & $(0.4,0.4)$ \\
\hline$\left(\vartheta_{3}, x, 1\right)$ & $(0.0,0.8)$ & $(0.3,0.3)$ & $(0.7,0.1)$ & $(0.2,0.1)$ & $(0.3,0.5)$ \\
\hline$\left(\vartheta_{3}, y, 1\right)$ & $(.05,0.8)$ & $(0.4,0.3)$ & $(0.5,0.1)$ & $(0.1, .15)$ & $(0.4,0.4)$ \\
\hline$\left(\vartheta_{3}, z, 1\right)$ & $(0.0,0.9)$ & $(0.3,0.4)$ & $(0.7, .05)$ & $(0.2,0.1)$ & $(0.5,0.4)$ \\
\hline$\left(\vartheta_{1}, x, 0\right)$ & $(0.8,0.1)$ & $(0.5,0.5)$ & $(0.1,0.8)$ & $(0.5,0.5)$ & $(0.5,0.4)$ \\
\hline$\left(\vartheta_{1}, y, 0\right)$ & $(0.9,0.1)$ & $(0.5,0.4)$ & $(0.2,0.8)$ & $(0.5,0.5)$ & $(0.5,0.4)$ \\
\hline$\left(\vartheta_{1}, z, 0\right)$ & $(0.8,0.2)$ & $(0.6,0.4)$ & $(0.2,0.7)$ & $(0.5,0.5)$ & $(0.4,0.4)$ \\
\hline$\left(\vartheta_{2}, x, 0\right)$ & $(1.0,0.0)$ & $(0.5,0.5)$ & $(0.1,0.8)$ & $(0.4,0.5)$ & $(0.5,0.5)$ \\
\hline$\left(\vartheta_{2}, y, 0\right)$ & $(0.9, .05)$ & $(0.4,0.5)$ & $(0.2,0.7)$ & $(0.5,0.4)$ & $(0.6, .35)$ \\
\hline$\left(\vartheta_{2}, z, 0\right)$ & $(0.9,0.1)$ & $(0.8,0.2)$ & $(0.3,0.6)$ & $(0.5,0.5)$ & $(0.5,0.4)$ \\
\hline$\left(\vartheta_{3}, x, 0\right)$ & $(0.8,0.1)$ & $(0.6,0.4)$ & $(0.1,0.7)$ & $(0.4,0.6)$ & $(0.6,0.3)$ \\
\hline$\left(\vartheta_{3}, y, 0\right)$ & $(0.8,0.1)$ & $(0.5,0.4)$ & $(0.3,0.5)$ & $(0.5,0.5)$ & $(0.5,0.4)$ \\
\hline$\left(\vartheta_{3}, z, 0\right)$ & $(0.9,0.0)$ & $(0.5,0.4)$ & $(0.2,0.7)$ & $(0.5,0.4)$ & $(0.4,0.5)$ \\
\hline
\end{tabular}

Table 4: FBSES $(\gamma, \chi, \mathscr{A})$.

\begin{tabular}{|c|c|c|c|c|c|}
\hline$(\gamma, \chi, \mathscr{A})$ & $u_{1}$ & $u_{2}$ & $u_{3}$ & $u_{4}$ & $u_{5}$ \\
\hline$\left(\vartheta_{1}, x, 1\right)$ & $(0.10,0.80)$ & $(0.30,0.40)$ & $(0.70,0.00)$ & $(0.20,0.20)$ & $(0.50,0.40)$ \\
\hline$\left(\vartheta_{2}, x, 0\right)$ & $(0.05,0.80)$ & $(0.40,0.30)$ & $(0.50,0.10)$ & $(0.10,0.15)$ & $(0.40,0.40)$ \\
\hline$\left(\vartheta_{3}, y, 1\right)$ & $(1.00,0.00)$ & $(0.50,0.50)$ & $(0.10,0.80)$ & $(0.40,0.50)$ & $(0.50,0.50)$ \\
\hline
\end{tabular}

TABle 5: FBSES $\left(\gamma_{1}, \chi_{1}, \mathscr{B}\right)$.

\begin{tabular}{|c|c|c|c|c|c|}
\hline$\left(\gamma_{1}, \chi_{1}, \mathscr{B}\right)$ & $u_{1}$ & $u_{2}$ & $u_{3}$ & $u_{4}$ & $u_{5}$ \\
\hline$\left(\vartheta_{1}, x, 1\right)$ & $(0.20,0.50)$ & $(0.40,0.20)$ & $(0.70,0.00)$ & $(0.20,0.10)$ & $(0.60,0.30)$ \\
\hline$\left(\vartheta_{1}, z, 1\right)$ & $(0.10,0.40)$ & $(0.50,0.30)$ & $(0.60,0.10)$ & $(0.10,0.20)$ & $(0.40,0.20)$ \\
\hline$\left(\vartheta_{2}, x, 0\right)$ & $(0.20,0.70)$ & $(0.50,0.20)$ & $(0.50,0.10)$ & $(0.20,0.10)$ & $(0.50,0.20)$ \\
\hline$\left(\vartheta_{3}, y, 1\right)$ & $(1.00,0.00)$ & $(0.60,0.40)$ & $(0.30,0.70)$ & $(0.50,0.30)$ & $(0.50,0.35)$ \\
\hline
\end{tabular}

TABle 6: $(\gamma, \chi, \mathscr{A})^{c}$.

\begin{tabular}{lcccrr}
\hline$(\gamma, \chi, \mathscr{A})^{c}$ & $u_{1}$ & $u_{2}$ & $u_{3}$ & $u_{4}$ & $u_{5}$ \\
\hline$\left(\vartheta_{1}, x, 1\right)$ & $(0.80,0.10)$ & $(0.40,0.30)$ & $(0.00,0.70)$ & $(0.20,0.20)$ & $(0.40,0.50)$ \\
$\left(\vartheta_{2}, x, 0\right)$ & $(0.80,0.05)$ & $(0.30,0.40)$ & $(0.10,0.50)$ & $(0.15,0.10)$ & $(0.40,0.40)$ \\
$\left(\vartheta_{3}, y, 1\right)$ & $(0.00,1.00)$ & $(0.50,0.50)$ & $(0.80,0.10)$ & $(0.50,0.40)$ & $(0.50,0.50)$ \\
\hline
\end{tabular}

Definition 9. A FBSES over the universe $\mathcal{U}$ is called a relative absolute FBSES, denoted by $(\mathfrak{U}, \Phi, \mathscr{A})$, if $\mathfrak{U}(\alpha)=\mathscr{U}$ (i.e., $(\mathcal{U}(\alpha))(u)=1, \forall \alpha \in \mathscr{A}$ and $u \in \mathscr{U})$ and $\Phi(\alpha)=\varnothing$ (i.e., $(\Phi(\alpha))(u)=0, \forall \alpha \in \mathscr{A}$ and $u \in \mathscr{U})$, for all $\alpha \in \mathscr{A}, \alpha \in \mathscr{A}$.

Definition 10. The support of a FBSES $(\gamma, \chi, \mathscr{A})$ on the universe $\mathcal{U}$ returns the objects $u \in \mathscr{U}$ with membership degrees greater than 0 . Mathematically,

$$
\begin{aligned}
& \operatorname{Supp}_{\gamma(\alpha)}=\{u \mid \gamma(\alpha)>0, \alpha \in \mathscr{A}\}, \\
& \operatorname{Supp}_{\chi(\alpha)}=\{u \mid \chi(\alpha)>0, \alpha \in \mathscr{A}\} .
\end{aligned}
$$

\section{Remark 1}

(i) $\operatorname{Supp}_{\gamma(\alpha)}=\mathscr{U}$ and $\operatorname{Supp}_{\chi(\alpha)}=\phi, \forall \alpha \in \mathscr{A}$ in the case of a relative absolute FBSES

(ii) $\operatorname{Supp}_{\gamma(\alpha)}=\phi$ and $\operatorname{Supp}_{\chi(\alpha)}=\mathscr{U}, \forall \alpha \in \mathscr{A}$ in the case of a relative null FBSES

Definition 11. An agree FBSES $(\gamma, \chi, \mathscr{A})_{1}$ on the universe $\mathscr{U}$ is a fuzzy bipolar soft expert subset of $(\gamma, \chi, \mathscr{A})$ given as

$$
(\gamma, \chi, \mathscr{A})_{1}=\left\{\gamma_{1}(\alpha), \chi_{1}(\alpha): \alpha \in \mathscr{V} \times E \times\{1\}\right\}
$$


Definition 12. A disagree FBSES $(\gamma, \chi, \mathscr{A})_{0}$ over the universe $\mathcal{U}$ is a fuzzy bipolar soft expert subset of $(\gamma, \chi, \mathscr{A})$ given by

$$
(\gamma, \chi, \mathscr{A})_{0}=\left\{\gamma_{0}(\alpha), \chi_{0}(\alpha): \alpha \in \mathscr{V} \times E \times\{0\}\right\} .
$$

Example 4. Consider the FBSES discussed in Example 1. Then, the agree FBSES and disagree FBSES are represented in Tables 7 and 8 , respectively.

Definition 13. For any two FBSESs $(\gamma, \chi, \mathscr{A})$ and $\left(\gamma_{1}, \chi_{1}, \mathscr{B}\right)$ over $\mathscr{U}$, “ $(\gamma, \chi, \mathscr{A})$ AND $\left(\gamma_{1}, \chi_{1}, \mathscr{B}\right)$ " denoted by $(\gamma, \chi, \mathscr{A}) \pi\left(\gamma_{1}, \chi_{1}, \mathscr{B}\right)$ is defined as

$$
(\gamma, \chi, \mathscr{A}) \pi\left(\gamma_{1}, \chi_{1}, \mathscr{B}\right)=(H, I, \mathscr{A} \times \mathscr{B}),
$$

where $H(\alpha, \beta)=\min \left(\gamma(\alpha), \gamma_{1}(\beta)\right)$ and $I(\alpha, \beta)=\max$ $\left(\chi(\alpha), \chi_{1}(\beta)\right), \forall(\alpha, \beta) \in \mathscr{A} \times \mathscr{B},(\alpha, \beta) \in \mathscr{A} \times \mathscr{B}$.

Example 5. Consider a company needs to fill a position, for which $\mathscr{U}=\left\{u_{1}, u_{2}, u_{3}, u_{4}\right\}$ represents the shortlisted candidates. Now the hiring board consisting of 2 members represented by $E=\{x, y\}$ considers $\mathscr{V}=\left\{\vartheta_{1}=\right.$ sound experience, $\vartheta_{2}=$ intelligence, $\vartheta_{3}=\operatorname{good}$ communication $\}$ and $\mathscr{V}=\left\{\vartheta_{1}=\right.$ little experience, $\vartheta_{2}=$ stupidity, $\vartheta_{3}=$ bad communication $\}$ as the decision parameters.

Let $\mathscr{A}=\left\{\left(\vartheta_{1}, x, 1\right),\left(\vartheta_{2}, y, 1\right),\left(\vartheta_{3}, x, 0\right)\right\}$ and $\mathscr{B}=\left\{\left(\vartheta_{1}, x\right.\right.$, $\left.0),\left(\vartheta_{2}, y, 1\right),\left(\vartheta_{3}, y, 1\right)\right\}$. Suppose $(\gamma, \chi, \mathscr{A})$ and $\left(\gamma_{1}, \chi_{1}, \mathscr{B}\right)$ are two FBSESs over $\mathcal{U}$ defined in Tables 9 and 10 , respectively.

Then, by Definition 13, the fuzzy bipolar soft expert AND operation between the above-defined FBSESs is given in Table 11.

Definition 14. For any two FBSESs $(\gamma, \chi, \mathscr{A})$ and $\left(\gamma_{1}, \chi_{1}, \mathscr{B}\right)$ over $\mathcal{U}$, “ $(\gamma, \chi, \mathscr{A})$ OR $\left(\gamma_{1}, \chi_{1}, \mathscr{B}\right)$ " denoted by $(\gamma, \chi, \mathscr{A}) \underline{\vee}\left(\gamma_{1}, \chi_{1}, \mathscr{B}\right)$ is defined as

$$
(\gamma, \chi, \mathscr{A}) \underline{\vee}\left(\gamma_{1}, \chi_{1}, \mathscr{B}\right)=(\stackrel{\circ}{H}, \stackrel{\circ}{I}, \mathscr{A} \times \mathscr{B}),
$$

where $\stackrel{H}{H}(\alpha, \beta)=\max \left(\gamma(\alpha), \gamma_{1}(\beta)\right)$ and $\stackrel{\circ}{I}(\alpha, \beta)=\min$ $\left(\chi(\alpha), \chi_{1}(\beta)\right), \forall(\alpha, \beta) \in \mathscr{A} \times \mathscr{B},(\alpha, \beta) \in \mathscr{A} \times \mathscr{B}$.

Proposition 2. Let $(\gamma, \chi, \mathscr{A})$ and $\left(\gamma_{1}, \chi_{1}, \mathscr{B}\right)$ be two FBSESs on $\mathcal{U}$, then

$$
\begin{aligned}
& \text { (1) }\left((\gamma, \chi, \mathscr{A}) \bar{\wedge}\left(\gamma_{1}, \chi_{1}, \mathscr{B}\right)\right)^{c}=(\gamma, \chi, \mathscr{A})^{c} \underline{\vee}\left(\gamma_{1}, \chi_{1}, \mathscr{B}\right)^{c} \\
& \text { (2) }\left((\gamma, \chi, \mathscr{A}) \underline{\mathrm{v}}\left(\gamma_{1}, \chi_{1}, \mathscr{B}\right)\right)^{c}=(\gamma, \chi, \mathscr{A})^{c} \bar{\wedge}\left(\gamma_{1}, \chi_{1}, \mathscr{B}\right)^{c}
\end{aligned}
$$

\section{Proof}

(1) Let $(\gamma, \chi, \mathscr{A}) \pi\left(\gamma_{1}, \chi_{1}, \mathscr{B}\right)=(J, K, \mathscr{A} \times \mathscr{B})$. Then for all $(\alpha, \beta) \in \mathscr{A} \times \mathscr{B}$,

$\left((\gamma, \chi, \mathscr{A}) \pi\left(\gamma_{1}, \chi_{1}, \mathscr{B}\right)\right)^{c}=(J, K, \mathscr{A} \times \mathscr{B})^{c}=\left(J^{c}, K^{c}, \mathscr{A} \times \mathscr{B}\right)$,

where $\quad J^{c}=\left(\min \left(\gamma(\alpha), \gamma_{1}(\beta)\right)\right)^{c}=\max \left(\gamma^{c}(\alpha)\right.$, $\left.\gamma_{1}^{c}(\beta)\right)$ and $K^{c}=\left(\max \left(\chi(\alpha), \chi_{1}(\beta)\right)\right)^{c}=\min \left(\chi^{c}(\alpha)\right.$, $\left.\chi_{1}^{\mathcal{c}}(\beta)\right)$.
TABle 7: Agree FBSES $(\gamma, \chi, X)_{1}$.

\begin{tabular}{lccccc}
\hline$(\gamma, \chi, X)_{1}$ & $u_{1}$ & $u_{2}$ & $u_{3}$ & $u_{4}$ & $u_{5}$ \\
\hline$\left(\vartheta_{1}, x\right)$ & $(0.1,0.8)$ & $(0.3,0.4)$ & $(0.7,0.0)$ & $(0.2,0.2)$ & $(0.5,0.4)$ \\
$\left(\vartheta_{1}, y\right)$ & $(0.1, .85)$ & $(0.4, .35)$ & $(0.8,0.1)$ & $(0.1,0.1)$ & $(0.4,0.5)$ \\
$\left(\vartheta_{1}, z\right)$ & $(0.2,0.7)$ & $(.25, .25)$ & $(0.7,0.2)$ & $(0.2,0.2)$ & $(0.4,0.4)$ \\
$\left(\vartheta_{2}, x\right)$ & $(0.0,0.6)$ & $(0.4,0.3)$ & $(0.8,0.0)$ & $(0.1,0.0)$ & $(0.3, .35)$ \\
$\left(\vartheta_{2}, y\right)$ & $(.05,0.7)$ & $(0.5,0.3)$ & $(0.7,0.0)$ & $(0.1, .05)$ & $(.35,0.4)$ \\
$\left(\vartheta_{2}, z\right)$ & $(0.0,0.6)$ & $(0.2, .25)$ & $(0.6,0.0)$ & $(.15,0.1)$ & $(0.4,0.4)$ \\
$\left(\vartheta_{3}, x\right)$ & $(0.0,0.8)$ & $(0.3,0.3)$ & $(0.7,0.1)$ & $(0.2,0.1)$ & $(0.3,0.5)$ \\
$\left(\vartheta_{3}, y\right)$ & $(.05,0.8)$ & $(0.4,0.3)$ & $(0.5,0.1)$ & $(0.1, .15)$ & $(0.4,0.4)$ \\
$\left(\vartheta_{3}, z\right)$ & $(0.0,0.9)$ & $(0.3,0.4)$ & $(0.7, .05)$ & $(0.2,0.1)$ & $(0.5,0.4)$ \\
\hline
\end{tabular}

TAble 8: Disagree FBSES $(\gamma, \chi, X)_{0}$.

\begin{tabular}{lccccc}
\hline$(\gamma, \chi, X)_{0}$ & $u_{1}$ & $u_{2}$ & $u_{3}$ & $u_{4}$ & $u_{5}$ \\
\hline$\left(\vartheta_{1}, x\right)$ & $(0.8,0.1)$ & $(0.5,0.5)$ & $(0.1,0.8)$ & $(0.5,0.5)$ & $(0.5,0.4)$ \\
$\left(\vartheta_{1}, y\right)$ & $(0.9,0.1)$ & $(0.5,0.4)$ & $(0.2,0.8)$ & $(0.5,0.5)$ & $(0.5,0.4)$ \\
$\left(\vartheta_{1}, z\right)$ & $(0.8,0.2)$ & $(0.6,0.4)$ & $(0.2,0.7)$ & $(0.5,0.5)$ & $(0.4,0.4)$ \\
$\left(\vartheta_{2}, x\right)$ & $(1.0,0.0)$ & $(0.5,0.5)$ & $(0.1,0.8)$ & $(0.4,0.5)$ & $(0.5,0.5)$ \\
$\left(\vartheta_{2}, y\right)$ & $(0.9, .05)$ & $(0.4,0.5)$ & $(0.2,0.7)$ & $(0.5,0.4)$ & $(0.6, .35)$ \\
$\left(\vartheta_{2}, z\right)$ & $(0.9,0.1)$ & $(0.8,0.2)$ & $(0.3,0.6)$ & $(0.5,0.5)$ & $(0.5,0.4)$ \\
$\left(\vartheta_{3}, x\right)$ & $(0.8,0.1)$ & $(0.6,0.4)$ & $(0.1,0.7)$ & $(0.4,0.6)$ & $(0.6,0.3)$ \\
$\left(\vartheta_{3}, y\right)$ & $(0.8,0.1)$ & $(0.5,0.4)$ & $(0.3,0.5)$ & $(0.5,0.5)$ & $(0.5,0.4)$ \\
$\left(\vartheta_{3}, z\right)$ & $(0.9,0.0)$ & $(0.5,0.4)$ & $(0.2,0.7)$ & $(0.5,0.4)$ & $(0.4,0.5)$ \\
\hline
\end{tabular}

Table 9: FBSES $(\gamma, \chi, \mathscr{A})$.

\begin{tabular}{ccccc}
\hline$(\gamma, \chi, \mathscr{A})$ & $u_{1}$ & $u_{2}$ & $u_{3}$ & $u_{4}$ \\
\hline$\left(\vartheta_{1}, x, 1\right)$ & $(0.70,0.10)$ & $(0.50,0.30)$ & $(0.90,0.00)$ & $(0.30,0.40)$ \\
$\left(\vartheta_{2}, y, 1\right)$ & $(0.80,0.10)$ & $(0.40,0.30)$ & $(0.80,0.10)$ & $(0.50,0.20)$ \\
$\left(\vartheta_{3}, x, 0\right)$ & $(0.30,0.50)$ & $(0.40,0.30)$ & $(0.20,0.60)$ & $(0.10,0.80)$ \\
\hline
\end{tabular}

TABle 10: FBSES $\left(\gamma_{1}, \chi_{1}, \mathscr{B}\right)$.

\begin{tabular}{lcccc}
\hline$\left(\gamma_{1}, \chi_{1}, \mathscr{B}\right)$ & $u_{1}$ & $u_{2}$ & $u_{3}$ & $u_{4}$ \\
\hline$\left(\vartheta_{2}, y, 1\right)$ & $(0.80,0.10)$ & $(0.40,0.30)$ & $(0.80,0.10)$ & $(0.50,0.20)$ \\
$\left(\vartheta_{3}, y, 1\right)$ & $(0.60,0.20)$ & $(0.40,0.30)$ & $(0.50,0.30)$ & $(0.80,0.10)$ \\
$\left(\vartheta_{1}, x, 0\right)$ & $(0.20,0.70)$ & $(0.40,0.50)$ & $(0.10,0.90)$ & $(0.60,0.40)$ \\
\hline
\end{tabular}

Now, let for all $(\alpha, \beta) \in \mathscr{A} \times \mathscr{B}$,

$$
(\gamma, \chi, \mathscr{A})^{c} \underline{\vee}\left(\gamma_{1}, \chi_{1}, \mathscr{B}\right)^{c}=(M, N, \mathscr{A} \times \mathscr{B}),
$$

where $\quad M=\max \left(\gamma^{c}(\alpha), \gamma_{1}^{c}(\beta)\right)$ and $\quad N=\min$ $\left(\chi^{c}(\alpha), \chi_{1}^{c}(\beta)\right)$.

Clearly, we have $M=J^{c}$ and $N=K^{c}$. Hence

$\left((\gamma, \chi, \mathscr{A}) \pi\left(\gamma_{1}, \chi_{1}, \mathscr{B}\right)\right)^{c}=(\gamma, \chi, \mathscr{A})^{c} \underline{\vee}\left(\gamma_{1}, \chi_{1}, \mathscr{B}\right)^{c}$.

(2) Let $(\gamma, \chi, \mathscr{A}) \underline{\vee}\left(\gamma_{1}, \chi_{1}, \mathscr{B}\right)=(J, K, \mathscr{A} \times \mathscr{B})$. Then, for all $(\alpha, \beta) \in \mathscr{A} \times \mathscr{B}$,

$\left((\gamma, \chi, \mathscr{A}) \underline{\vee}\left(\gamma_{1}, \chi_{1}, \mathscr{B}\right)\right)^{c}=(J, K, \mathscr{A} \times \mathscr{B})^{c}=\left(J^{c}, K^{c}, \mathscr{A} \times \mathscr{B}\right)$,

where $\quad J^{c}=\left(\max \left(\gamma(\alpha), \gamma_{1}(\beta)\right)\right)^{c}=\min \left(\gamma^{c}(\alpha), \gamma_{1}^{c}\right.$ $(\beta))$ and $K^{c}=\left(\min \left(\chi(\alpha), \chi_{1}(\beta)\right)\right)^{c}=\max \left(\chi^{c}(\alpha)\right.$, $\left.\chi_{1}^{c}(\beta)\right)$. 
TABLE 11: AND operation between FBSESs $(\gamma, \chi, \mathscr{A})$ and $\left(\gamma_{1}, \chi_{1}, \mathscr{B}\right)$.

\begin{tabular}{lccrr}
\hline$(H, I, \mathscr{A} \times \mathscr{B})$ & $u_{1}$ & $u_{2}$ & $u_{3}$ & $u_{4}$ \\
\hline$\left(\vartheta_{1}, x, 1\right) \pi\left(\vartheta_{2}, y, 1\right)$ & $(0.70,0.10)$ & $(0.40,0.30)$ & $(0.80,0.10)$ & $(0.30,0.40)$ \\
$\left(\vartheta_{1}, x, 1\right) \pi\left(\vartheta_{3}, y, 1\right)$ & $(0.60,0.20)$ & $(0.40,0.30)$ & $(0.50,0.30)$ & $(0.30,0.40)$ \\
$\left(\vartheta_{1}, x, 1\right) \pi\left(\vartheta_{1}, x, 0\right)$ & $(0.20,0.70)$ & $(0.40,0.50)$ & $(0.10,0.90)$ & $(0.30,0.40)$ \\
$\left(\vartheta_{2}, y, 1\right) \pi\left(\vartheta_{2}, y, 1\right)$ & $(0.80,0.10)$ & $(0.40,0.30)$ & $(0.50,0.30)$ & $(0.50,0.20)$ \\
$\left(\vartheta_{2}, y, 1\right) \pi\left(\vartheta_{3}, y, 1\right)$ & $(0.60,0.20)$ & $(0.40,0.30)$ & $(0.10,0.90)$ & $(0.50,0.20)$ \\
$\left(\vartheta_{2}, y, 1\right) \pi\left(\vartheta_{1}, x, 0\right)$ & $(0.20,0.70)$ & $(0.40,0.50)$ & $(0.20,0.60)$ & $(0.50,0.40)$ \\
$\left(\vartheta_{3}, x, 0\right) \pi\left(\vartheta_{2}, y, 1\right)$ & $(0.30,0.50)$ & $(0.40,0.30)$ & $(0.20,0.60)$ & $(0.10,0.80)$ \\
$\left(\vartheta_{3}, x, 0\right) \pi\left(\vartheta_{3}, y, 1\right)$ & $(0.30,0.50)$ & $(0.40,0.30)$ & $(0.10,0.90)$ & $(0.10,0.80)$ \\
$\left(\vartheta_{3}, x, 0\right) \pi\left(\vartheta_{1}, x, 0\right)$ & $(0.20,0.70)$ & $(0.40,0.50)$ & & \\
\hline
\end{tabular}

Now, let for all $(\alpha, \beta) \in \mathscr{A} \times \mathscr{B}$,

$$
(\gamma, \chi, \mathscr{A})^{c} \pi\left(\gamma_{1}, \chi_{1}, \mathscr{B}\right)^{c}=(M, N, \mathscr{A} \times \mathscr{B}) .
$$

where $\quad M=\min \left(\gamma^{c}(\alpha), \gamma_{1}^{c}(\beta)\right)$ and $\quad N=\max$ $\left(\chi^{c}(\alpha), \chi_{1}^{c}(\beta)\right)$

Clearly, we have $M=J^{c}$ and $N=K^{c}$. Hence

$\left((\gamma, \chi, \mathscr{A}) \underline{\vee}\left(\gamma_{1}, \chi_{1}, \mathscr{B}\right)\right)^{c}=(\gamma, \chi, \mathscr{A})^{c} \pi\left(\gamma_{1}, \chi_{1}, \mathscr{B}\right)^{c}$.

Example 6. Consider the FBSESs discussed in Example 5. By Definition 14, the fuzzy bipolar soft expert OR operation on these sets (i.e., $\left.(\gamma, \chi, \mathscr{A}) \underline{\vee}\left(\gamma_{1}, \chi_{1}, \mathscr{B}\right)=(\stackrel{\circ}{H}, \stackrel{\circ}{I}, \mathscr{A} \times \mathscr{B})\right)$ is given in Table 12.

Proposition 3. If $\left(\gamma_{1}, \chi_{1}, \mathscr{A}\right),\left(\gamma_{2}, \chi_{2}, \mathscr{B}\right)$, and $\left(\gamma_{3}, \chi_{3}, \mathscr{C}\right)$ are 3 FBSESs over the universe $\mathcal{U}$, then
(1) $\left(\gamma_{1}, \chi_{1}, \mathscr{A}\right) \underline{v}\left(\left(\gamma_{2}, \chi_{2}, \mathscr{B}\right) \underline{\vee}\left(\gamma_{3}, \chi_{3}, \mathscr{C}\right)\right)=\left(\left(\gamma_{1}, \chi_{1}, \mathscr{A}\right)\right.$ $\left.\underline{\mathrm{v}}\left(\gamma_{2}, \chi_{2}, \mathscr{B}\right)\right) \underline{\mathrm{v}}\left(\gamma_{3}, \chi_{3}, \mathscr{C}\right)$

(2) $\left(\gamma_{1}, \chi_{1}, \mathscr{A}\right) \pi\left(\left(\gamma_{2}, \chi_{2}, \mathscr{B}\right) \pi\left(\gamma_{3}, \chi_{3}, \mathscr{C}\right)\right)=\left(\left(\gamma_{1}, \chi_{1}, \mathscr{A}\right)\right.$ $\left.\bar{\Lambda}\left(\gamma_{2}, \chi_{2}, \mathscr{B}\right)\right) \bar{\lambda}\left(\gamma_{3}, \chi_{3}, \mathscr{C}\right)$

(3) $\left(\gamma_{1}, \chi_{1}, \mathscr{A}\right) \underline{\vee}\left(\left(\gamma_{2}, \chi_{2}, \mathscr{B}\right) \pi\left(\gamma_{3}, \chi_{3}, \mathscr{C}\right)\right)=\left(\left(\gamma_{1}, \chi_{1}, \mathscr{A}\right)\right.$ $\left.\left.\underline{\vee}\left(\gamma_{2}, \chi_{2}, \mathscr{B}\right)\right) \pi\left(\left(\gamma_{1}, \chi_{1}, \mathscr{B}\right) \underline{\mathrm{v}}\left(\gamma_{3}, \chi_{3}, \mathscr{C}\right)\right)\right)$

(4) $\left(\gamma_{1}, \chi_{1}, \mathscr{A}\right) \pi\left(\left(\gamma_{2}, \chi_{2}, \mathscr{B}\right) \underline{\vee}\left(\gamma_{3}, \chi_{3}, \mathscr{C}\right)\right)=\left(\left(\gamma_{1}, \chi_{1}, \mathscr{A}\right)\right.$ $\left.\bar{\wedge}\left(\gamma_{2}, \chi_{2}, \mathscr{B}\right)\right) \underline{v}\left(\left(\gamma_{1}, \chi_{1}, \mathscr{B}\right) \pi\left(\gamma_{3}, \chi_{3}, \mathscr{C}\right)\right)$

Proof

(1) Suppose that $\gamma_{2}(\beta) \underline{v} \gamma_{3}(\lambda)=\max \left(\gamma_{2}(\beta), \quad \gamma_{3}(\lambda)\right)$, $\forall(\beta, \lambda) \in \mathscr{B} \times \mathscr{C}$.

Then,

$$
\begin{aligned}
\gamma_{1}(\alpha) \underline{\vee}\left(\gamma_{2}(\beta) \underline{\vee} \gamma_{3}(\lambda)\right) & =\max \left(\gamma_{1}(\alpha), \max \left(\gamma_{2}(\beta), \gamma_{3}(\lambda)\right)\right), \quad \forall(\alpha,(\beta, \lambda)) \in \mathscr{A} \times \mathscr{B} \times \mathscr{C} \\
& =\max \left(\max \left(\gamma_{1}(\alpha), \gamma_{2}(\beta)\right), \gamma_{3}(\lambda)\right), \quad \forall((\alpha, \beta), \lambda) \in \mathscr{A} \times \mathscr{B} \times \mathscr{C} \\
& =\left(\gamma_{1}(\alpha) \underline{\vee} \gamma_{2}(\beta)\right) \underline{\vee}\left(\gamma_{3}(\lambda)\right) .
\end{aligned}
$$

Again suppose that $\chi_{2}(\beta) \underline{\vee} \chi_{3}(\lambda)=\min \left(\chi_{2}(\beta), \chi_{3}\right.$

Then,

$(\lambda)), \quad \forall(\beta, \lambda) \in \mathscr{B} \times \mathscr{C}$.

$$
\begin{aligned}
\chi_{1}(\alpha) \underline{v}\left(\chi_{2}(\beta) \underline{\vee} \chi_{3}(\lambda)\right) & =\min \left(\chi_{1}(\alpha), \min \left(\chi_{2}(\beta), \chi_{3}(\lambda)\right)\right), \quad \forall(\alpha,(\beta, \lambda)) \in \mathscr{A} \times \mathscr{B} \times \mathscr{C} \\
& =\min \left(\min \left(\chi_{1}(\alpha), \chi_{2}(\beta)\right), \chi_{3}(\lambda)\right), \quad \forall((\alpha, \beta), \lambda) \in \mathscr{A} \times \mathscr{B} \times \mathscr{C} \\
& =\left(\chi_{1}(\alpha) \underline{v} \chi_{2}(\beta)\right) \underline{v}\left(\chi_{3}(\lambda)\right) .
\end{aligned}
$$

Hence $\quad\left(\gamma_{1}, \chi_{1}, \mathscr{A}\right) \underline{\vee}\left(\left(\gamma_{2}, \quad \chi_{2}, \mathscr{B}\right) \underline{\vee}\left(\gamma_{3}, \chi_{3}, \mathscr{C}\right)\right)=$ $\left(\left(\gamma_{1}, \chi_{1}, \mathscr{A}\right) \underline{\vee}\left(\gamma_{2}, \chi_{2}, \mathscr{B}\right)\right) \underline{\vee}\left(\gamma_{3}, \chi_{3}, \mathscr{C}\right)$.
(2) Suppose that $\gamma_{2}(\beta) \bar{\wedge} \gamma_{3}(\lambda)=\min \left(\gamma_{2}(\beta), \gamma_{3}\right.$ $(\lambda)), \quad \forall(\beta, \lambda) \in \mathscr{B} \times \mathscr{C}$. 
TABLE 12: OR operation between FBSESs $(\gamma, \chi, \mathscr{A})$ and $\left(\gamma_{1}, \chi_{1}, \mathscr{B}\right)$.

\begin{tabular}{|c|c|c|c|c|}
\hline$(\stackrel{H}{H}, \stackrel{\mathscr{A}}{A} \times \mathscr{B})$ & $u_{1}$ & $u_{2}$ & $u_{3}$ & $u_{4}$ \\
\hline$\left(\vartheta_{1}, x, 1\right) \underline{v}\left(\vartheta_{2}, y, 1\right)$ & $(0.80,0.10)$ & $(0.50,0.30)$ & $(0.90,0.00)$ & $(0.50,0.20)$ \\
\hline$\left(\vartheta_{1}, x, 1\right) \unrhd\left(\vartheta_{3}, y, 1\right)$ & $(0.70,0.10)$ & $(0.50,0.30)$ & $(0.90,0.00)$ & $(0.80,0.10)$ \\
\hline$\left(\vartheta_{1}, x, 1\right) \underline{\vee}\left(\vartheta_{1}, x, 0\right)$ & $(0.70,0.10)$ & $(0.50,0.30)$ & $(0.90,0.00)$ & $(0.60,0.40)$ \\
\hline$\left(\vartheta_{2}, y, 1\right) \underline{\vee}\left(\vartheta_{2}, y, 1\right)$ & $(0.80,0.10)$ & $(0.40,0.30)$ & $(0.80,0.10)$ & $(0.50,0.20)$ \\
\hline$\left(\vartheta_{2}, y, 1\right) \vee\left(\vartheta_{3}, y, 1\right)$ & $(0.80,0.10)$ & $(0.40,0.30)$ & $(0.80,0.10)$ & $(0.80,0.10)$ \\
\hline$\left(\vartheta_{2}, y, 1\right) \vee\left(\vartheta_{1}, x, 0\right)$ & $(0.80,0.10)$ & $(0.40,0.30)$ & $(0.80,0.10)$ & $(0.60,0.20)$ \\
\hline$\left(\vartheta_{3}, x, 0\right) \underline{\vee}\left(\vartheta_{2}, y, 1\right)$ & $(0.80,0.10)$ & $(0.40,0.30)$ & $(0.80,0.10)$ & $(0.50,0.20)$ \\
\hline$\left(\vartheta_{3}, x, 0\right) \underline{\vee}\left(\vartheta_{3}, y, 1\right)$ & $(0.60,0.20)$ & $(0.40,0.30)$ & $(0.50,0.30)$ & $(0.80,0.10)$ \\
\hline$\left(\vartheta_{3}, x, 0\right) \underline{v}\left(\vartheta_{1}, x, 0\right)$ & $(0.30,0.50)$ & $(0.40,0.30)$ & $(0.20,0.60)$ & $(0.60,0.40)$ \\
\hline
\end{tabular}

Then,

$$
\begin{aligned}
\gamma_{1}(\alpha) \pi\left(\gamma_{2}(\beta) \pi \gamma_{3}(\lambda)\right) & =\min \left(\gamma_{1}(\alpha), \min \left(\gamma_{2}(\beta), \gamma_{3}(\lambda)\right)\right), \quad \forall(\alpha,(\beta, \lambda)) \in \mathscr{A} \times \mathscr{B} \times \mathscr{C} \\
& =\min \left(\min \left(\gamma_{1}(\alpha), \gamma_{2}(\beta)\right), \gamma_{3}(\lambda)\right), \quad \forall((\alpha, \beta), \lambda) \in \mathscr{A} \times \mathscr{B} \times \mathscr{C} \\
& =\left(\gamma_{1}(\alpha) \pi \gamma_{2}(\beta)\right) \pi\left(\gamma_{3}(\lambda)\right) .
\end{aligned}
$$

Again suppose that $\chi_{2}(\beta) \pi \chi_{3}(\lambda)=\max \left(\chi_{2}(\beta), \quad\right.$ Then,

$\left.\chi_{3}(\lambda)\right), \quad \forall(\beta, \lambda) \in \mathscr{B} \times \mathscr{C}$.

$$
\begin{aligned}
\chi_{1}(\alpha) \pi\left(\chi_{2}(\beta) \pi \chi_{3}(\lambda)\right) & =\max \left(\chi_{1}(\alpha), \max \left(\chi_{2}(\beta), \chi_{3}(\lambda)\right)\right), \quad \forall(\alpha,(\beta, \lambda)) \in \mathscr{A} \times \mathscr{B} \times \mathscr{C} \\
& =\max \left(\max \left(\chi_{1}(\alpha), \chi_{2}(\beta)\right), \chi_{3}(\lambda)\right), \quad \forall((\alpha, \beta), \lambda) \in \mathscr{A} \times \mathscr{B} \times \mathscr{C} \\
& =\left(\chi_{1}(\alpha) \pi \chi_{2}(\beta)\right) \pi\left(\chi_{3}(\lambda)\right) .
\end{aligned}
$$

Hence $\quad\left(\gamma_{1}, \chi_{1}, \mathscr{A}\right) \pi\left(\left(\gamma_{2}, \chi_{2}, \mathscr{B}\right) \pi\left(\gamma_{3}\right.\right.$, $\left.\left.\chi_{3}, \mathscr{C}\right)\right)=\left(\left(\gamma_{1}, \chi_{1}, \mathscr{A}\right) \pi\left(\gamma_{2}, \chi_{2}, \mathscr{B}\right)\right) \pi\left(\gamma_{3}, \chi_{3}, \mathscr{C}\right)$.
(3) Suppose that $\gamma_{2}(\beta) \pi \gamma_{3}(\lambda)=\min \left(\gamma_{2}(\beta), \gamma_{3}\right.$ $(\lambda)), \quad \forall(\beta, \lambda) \in \mathscr{B} \times \mathscr{C}$.

Then,

$$
\begin{aligned}
\gamma_{1}(\alpha) \underline{\vee}\left(\gamma_{2}(\beta) \pi \gamma_{3}(\lambda)\right) & =\max \left(\gamma_{1}(\alpha), \min \left(\gamma_{2}(\beta), \gamma_{3}(\lambda)\right)\right), \quad \forall(\alpha,(\beta, \lambda)) \in \mathscr{A} \times \mathscr{B} \times \mathscr{C} \\
& =\min \left(\max \left(\gamma_{1}(\alpha), \gamma_{2}(\beta)\right), \max \left(\gamma_{1}(\alpha), \gamma_{3}(\lambda)\right)\right) \\
& =\left(\gamma_{1}(\alpha) \underline{\vee} \gamma_{2}(\beta)\right) \pi\left(\gamma_{1}(\alpha) \underline{\vee} \gamma_{3}(\lambda)\right) .
\end{aligned}
$$

Now suppose that $\chi_{2}(\beta) \pi \chi_{3}(\lambda)=\max \left(\chi_{2}(\beta)\right.$, $\left.\chi_{3}(\lambda)\right), \quad \forall(\beta, \lambda) \in \mathscr{B} \times \mathscr{C}$.
Then,

$$
\begin{aligned}
\chi_{1}(\alpha) \underline{\vee}\left(\chi_{2}(\beta) \pi \chi_{3}(\lambda)\right) & =\min \left(\chi_{1}(\alpha), \max \left(\chi_{2}(\beta), \chi_{3}(\lambda)\right)\right), \quad \forall(\alpha,(\beta, \lambda)) \in \mathscr{A} \times \mathscr{B} \times \mathscr{C} \\
& =\max \left(\min \left(\chi_{1}(\alpha), \chi_{2}(\beta)\right), \min \left(\chi_{1}(\alpha), \chi_{3}(\lambda)\right)\right) \\
& =\left(\chi_{1}(\alpha) \underline{\vee} \chi_{2}(\beta)\right) \pi\left(\chi_{1}(\alpha) \underline{\vee} \chi_{3}(\lambda)\right) .
\end{aligned}
$$

Hence, $\quad\left(\gamma_{1}, \chi_{1}, \mathscr{A}\right) \underline{\vee}\left(\left(\gamma_{2}, \chi_{2}, \mathscr{B}\right) \quad \pi\left(\gamma_{3}, \chi_{3}, \mathscr{C}\right)\right)=$ $\left(\left(\gamma_{1}, \chi_{1}, \mathscr{A}\right) \underline{\vee}\left(\gamma_{2}, \chi_{2}, \mathscr{B}\right)\right)$

$\bar{\Lambda}\left(\left(\gamma_{1}, \chi_{1}, \mathscr{B}\right) \underline{\vee}\left(\gamma_{3}, \chi_{3}, \mathscr{C}\right)\right)$.

(4) Similar to 3.
Definition 15. Extended fuzzy union of any two FBSESs $(\gamma, \chi, \mathscr{A})$ and $\left(\gamma_{1}, \chi_{1}, \mathscr{B}\right)$ over the same universe $\mathcal{U}$ results in a new FBSES $\left(H_{\cup}, I_{\cap}, C\right)$ over $\mathcal{U}$, where $C=\mathscr{A} \cup \mathscr{B}$ and for all $\alpha \in C$, 


$$
\begin{aligned}
& H_{\cup}(\alpha)= \begin{cases}\gamma(\alpha), & \text { if } \alpha \in \mathscr{A}-\mathscr{B}, \\
\gamma_{1}(\alpha), & \text { if } \alpha \in \mathscr{B}-\mathscr{A}, \\
\max \left(\gamma(\alpha), \gamma_{1}(\alpha)\right), & \text { if } \alpha \in \mathscr{A} \cap \mathscr{B},\end{cases} \\
& I_{\cap}(\alpha)= \begin{cases}\chi(\alpha), & \text { if } \alpha \in(\mathscr{A})-(\mathscr{B}), \\
\chi_{1}(\alpha), & \text { if } \alpha \in(\mathscr{B})-(\mathscr{A}), \\
\min \left(\chi(\alpha), \chi_{1}(\alpha)\right), & \text { if } \alpha \in(\mathscr{A}) \cap(\mathscr{B}) .\end{cases}
\end{aligned}
$$

We denote it by $(\gamma, \chi, \mathscr{A}) \widehat{\cup}\left(\gamma_{1}, \chi_{1}, \mathscr{B}\right)=\left(H_{\cup}, I_{\cap}, C\right)$.

Example 7. A business corporation decides to boost its business by using an AI virtual chatbot to reply the queries of their customers more efficiently and make better integration with all forums. The corporation assigns the task to two experienced virtual assistants to compare the available AI chatbots in order to find the best one for their needs.

The set $\mathcal{U}=\left\{u_{1}, u_{2}, u_{3}, u_{4}, u_{5}\right\}$ represents the available competing AI virtual chatbots. The set $\mathscr{V}=\left\{\vartheta_{1}=\right.$ quick response, $\vartheta_{2}=$ powerful integration, $\vartheta_{3}=$ comprehension $\}$ represents the suitable parameters; then, $\mathscr{V}=\left\{\vartheta_{1}=\right.$ loose response, $\vartheta_{2}=$ weak integration, $\vartheta_{3}=$ misinterpretation $\}$. Let $E=\{x, y\}$ represent the set of experts and $\mathcal{O}=\{1=$ agree, $0=$ disagree $\}$ represent the set of opinions.

Let $\mathscr{A}=\left\{\left(\vartheta_{1}, x, 1\right),\left(\vartheta_{2}, x, 1\right),\left(\vartheta_{3}, y, 0\right)\right\} \quad$ and $\mathscr{B}=\left\{\left(\vartheta_{1}, x, 1\right),\left(\vartheta_{1}, y, 1\right),\left(\vartheta_{2}, x, 1\right)\right\}$. Now suppose that we have the FBSESs $(\gamma, \chi, \mathscr{A})$ and $\left(\gamma_{1}, \chi_{1}, \mathscr{B}\right)$ defined in Tables 13 and 14 , respectively.

Then their extended union $\left(H_{\cup}, I_{\cap}, C\right)$, where $C=\mathscr{A} \cup \mathscr{B}$, is defined in Table 15 .

Definition 16. Extended fuzzy intersection of two FBSESs $(\gamma, \chi, \mathscr{A})$ and $\left(\gamma_{1}, \chi_{1}, \mathscr{B}\right)$ on $\mathcal{U}$ results in a new FBSES $\left(H_{\cap}, I_{\cup}, C\right)$ over $\mathcal{U}$, where $C=\mathscr{A} \cup \mathscr{B}$ and $\forall \alpha \in C$,

$$
\begin{aligned}
H_{\cap}(\alpha) & = \begin{cases}\gamma(\alpha), & \text { if } \alpha \in \mathscr{A}-\mathscr{B}, \\
\gamma_{1}(\alpha), & \text { if } \alpha \in \mathscr{B}-\mathscr{A}, \\
\min \left(\gamma(\alpha), \gamma_{1}(\alpha)\right), & \text { if } \alpha \in \mathscr{A} \cap \mathscr{B},\end{cases} \\
I_{\cup}(\alpha) & = \begin{cases}\chi(\alpha), & \text { if } \alpha \in(\mathscr{A})-(\mathscr{B}), \\
\chi_{1}(\alpha), & \text { if } \alpha \in(\mathscr{B})-(\mathscr{A}), \\
\max \left(\chi(\alpha), \chi_{1}(\alpha)\right), & \text { if } \alpha \in(\mathscr{A}) \cap(\mathscr{B}) .\end{cases}
\end{aligned}
$$

We denote it by $(\gamma, \chi, \mathscr{A}) \widehat{\cap}\left(\gamma_{1}, \chi_{1}, \mathscr{B}\right)=\left(H_{\cap}, I_{\cup}, C\right)$.

Example 8. Consider the FBSESs $(\gamma, \chi, \mathscr{A})$ and $\left(\gamma_{1}, \chi_{1}, \mathscr{B}\right)$ in Example 7. Their extended intersection $\left(H_{\cap}, I_{\cup}, C\right)$, where $C=\mathscr{A} \times \mathscr{B}$, is defined in Table 16 .

Proposition 4. If $\left(\gamma_{1}, \chi_{1}, \mathscr{A}\right),\left(\gamma_{2}, \chi_{2}, \mathscr{B}\right)$, and $\left(\gamma_{3}, \chi_{3}, \mathscr{C}\right)$ are three FBSESs on universe $\mathcal{U}$, then

(1) $\left(\gamma_{1}, \chi_{1}, \mathscr{A}\right) \widehat{U}\left(\gamma_{2}, \chi_{2}, \mathscr{B}\right)=\left(\gamma_{2}, \chi_{2}, \mathscr{B}\right) \widehat{U}\left(\gamma_{1}, \chi_{1}, \mathscr{A}\right)$

(2) $\left(\gamma_{1}, \chi_{1}, \mathscr{A}\right) \widehat{\cap}\left(\gamma_{2}, \chi_{2}, \mathscr{B}\right)=\left(\gamma_{2}, \chi_{2}, \mathscr{B}\right) \widehat{\cap}\left(\gamma_{1}, \chi_{1}, \mathscr{A}\right)$

(3) $\left(\gamma_{1}, \chi_{1}, \mathscr{A}\right) \widehat{U}\left(\left(\gamma_{2}, \chi_{2}, \mathscr{B}\right) \widehat{U}\left(\gamma_{3}, \chi_{3}, \mathscr{C}\right)\right)=\left(\left(\gamma_{1}\right.\right.$, $\left.\left.\chi_{1}, \mathscr{A}\right) \widehat{U}\left(\gamma_{2}, \chi_{2}, \mathscr{B}\right)\right) \widehat{U}\left(\gamma_{3}, \chi_{3}, \mathscr{C}\right)$

(4) $\left(\gamma_{1}, \chi_{1}, \mathscr{A}\right) \widehat{\cap}\left(\left(\gamma_{2}, \chi_{2}, \quad \mathscr{B}\right) \widehat{\cap}\left(\gamma_{3}, \chi_{3}, \mathscr{C}\right)\right)=\left(\left(\gamma_{1}, \chi_{1}\right.\right.$, $\left.\mathscr{A}) \widehat{\cap}\left(\gamma_{2}, \chi_{2}, \mathscr{B}\right)\right) \hat{\cap}\left(\gamma_{3}, \chi_{3}, \mathscr{C}\right)$

Proof. (1) Let $\left(\gamma_{1}, \chi_{1}, \mathscr{A}\right) \widehat{\cup}\left(\gamma_{2}, \chi_{2}, \mathscr{B}\right)=(P, Q, \mathscr{A} \cup \mathscr{B})$. From Definition 15, for all $\alpha \in \mathscr{A} \cup \mathscr{B}$, we have

$$
\begin{gathered}
P(\alpha)= \begin{cases}\gamma_{1}(\alpha), & \text { if } \alpha \in \mathscr{A}-\mathscr{B}, \\
\gamma_{2}(\alpha), & \text { if } \alpha \in \mathscr{B}-\mathscr{A}, \\
\max \left(\gamma_{1}(\alpha), \gamma_{2}(\alpha)\right), & \text { if } \alpha \in \mathscr{A} \cap \mathscr{B},\end{cases} \\
Q(\alpha)= \begin{cases}\chi_{1}(\alpha), & \text { if } \alpha \in(\mathscr{A})-(\mathscr{B}), \\
\chi_{2}(\alpha), & \text { if } \alpha \in(\mathscr{B})-(\mathscr{A}), \\
\min \left(\chi_{1}(\alpha), \chi_{2}(\alpha)\right), & \text { if } \alpha \in(\mathscr{A}) \cap(\mathscr{B}) .\end{cases}
\end{gathered}
$$

We consider only the nontrivial case when $\alpha \in \mathscr{A} \cap \mathscr{B}$, and then we have

$$
\gamma_{1}(\alpha) \widehat{U} \gamma_{2}(\alpha)=P(\alpha)=\max \left(\gamma_{1}(\alpha), \gamma_{2}(\alpha)\right)=\max \left(\gamma_{2}(\alpha), \gamma_{1}(\alpha)\right)=\gamma_{2}(\alpha) \widehat{U} \gamma_{1}(\alpha)
$$

Similarly,

$$
\chi_{1}(\alpha) \widehat{U} \chi_{2}(\alpha)=Q(\alpha)=\min \left(\chi_{1}(\alpha), \chi_{2}(\alpha)\right)=\min \left(\chi_{2}(\alpha), \chi_{1}(\alpha)\right)=\chi_{2}(\alpha) \widehat{U} \chi_{1}(\alpha)
$$


TABle 13: $(\gamma, \chi, \mathscr{A})$.

\begin{tabular}{lcccrr}
\hline$(\gamma, \chi, \mathscr{A})$ & $u_{1}$ & $u_{2}$ & $u_{3}$ & $u_{4}$ & $u_{5}$ \\
\hline$\left(\vartheta_{1}, x, 1\right)$ & $(0.40,0.30)$ & $(0.50,0.20)$ & $(0.80,0.10)$ & $(0.30,0.40)$ & $(0.70,0.10)$ \\
$\left(\vartheta_{2}, x, 1\right)$ & $(0.50,0.25)$ & $(0.60,0.20)$ & $(0.80,0.00)$ & $(0.55,0.30)$ & $(0.30,0.50)$ \\
$\left(\vartheta_{3}, y, 0\right)$ & $(0.40,0.50)$ & $(0.30,0.50)$ & $(0.30,0.40)$ & $(0.50,0.40)$ & $(0.20,0.60)$ \\
\hline
\end{tabular}

TABLE 14: $\left(\gamma_{1}, \chi_{1}, B\right)$.

\begin{tabular}{|c|c|c|c|c|c|}
\hline$\left(\gamma_{1}, \chi_{1}, B\right)$ & $u_{1}$ & $u_{2}$ & $u_{3}$ & $u_{4}$ & $u_{5}$ \\
\hline$\left(\vartheta_{1}, x, 1\right)$ & $(0.50,0.40)$ & $(0.50,0.30)$ & $(0.70,0.20)$ & $(0.40,0.30)$ & $(0.80,0.10)$ \\
\hline$\left(\vartheta_{1}, y, 1\right)$ & $(0.40,0.50)$ & $(0.60,0.20)$ & $(0.80,0.10)$ & $(0.40,0.40)$ & $(0.70,0.20)$ \\
\hline$\left(\vartheta_{2}, x, 1\right)$ & $(0.50,0.40)$ & $(0.50,0.20)$ & $(0.80,0.10)$ & $(0.50,0.30)$ & $(0.40,0.40)$ \\
\hline
\end{tabular}

TABle 15: $\left(H_{\cup}, I_{\cap}, C\right)$.

\begin{tabular}{|c|c|c|c|c|c|}
\hline$\left(H_{\cup}, I_{\cap}, C\right)$ & $u_{1}$ & $u_{2}$ & $u_{3}$ & $u_{4}$ & $u_{5}$ \\
\hline$\left(\vartheta_{1}, x, 1\right)$ & $(0.50,0.30)$ & $(0.50,0.20)$ & $(0.80,0.10)$ & $(0.40,0.30)$ & $(0.80,0.10)$ \\
\hline$\left(\vartheta_{1}, y, 1\right)$ & $(0.40,0.50)$ & $(0.60,0.20)$ & $(0.80,0.10)$ & $(0.40,0.40)$ & $(0.70,0.20)$ \\
\hline$\left(\vartheta_{2}, x, 1\right)$ & $(0.50,0.20)$ & $(0.60,0.20)$ & $(0.80,0.00)$ & $(0.50,0.30)$ & $(0.40,0.40)$ \\
\hline$\left(\vartheta_{3}, y, 0\right)$ & $(0.40,0.50)$ & $(0.30,0.50)$ & $(0.30,0.40)$ & $(0.50,0.40)$ & $(0.20,0.60)$ \\
\hline
\end{tabular}

TABle 16: $\left(H_{\cap}, I_{\cup}, C\right)$.

\begin{tabular}{|c|c|c|c|c|c|}
\hline$\left(H_{\cap}, I_{\cup}, C\right)$ & $u_{1}$ & $u_{2}$ & $u_{3}$ & $u_{4}$ & $u_{5}$ \\
\hline$\left(\vartheta_{1}, x, 1\right)$ & $(0.40,0.40)$ & $(0.50,0.30)$ & $(0.70,0.20)$ & $(0.30,0.40)$ & $(0.70,0.10)$ \\
\hline$\left(\vartheta_{1}, y, 1\right)$ & $(0.40,0.50)$ & $(0.60,0.20)$ & $(0.80,0.10)$ & $(0.40,0.40)$ & $(0.70,0.20)$ \\
\hline$\left(\vartheta_{2}, x, 1\right)$ & $(0.50,0.40)$ & $(0.50,0.20)$ & $(0.80,0.10)$ & $(0.50,0.30)$ & $(0.30,0.50)$ \\
\hline$\left(\vartheta_{3}, y, 0\right)$ & $(0.40,0.50)$ & $(0.30,0.50)$ & $(0.30,0.40)$ & $(0.50,0.40)$ & $(0.20,0.60)$ \\
\hline
\end{tabular}

TABLe 17: Restricted union $\left(H_{\cup}^{\prime}, I_{\cap}^{\prime}, C\right)$.

\begin{tabular}{lcccrr}
\hline$\left(H_{\cup}^{\prime}, I_{\cap}^{\prime}, C\right)$ & $u_{1}$ & $u_{2}$ & $u_{3}$ & $u_{4}$ & $u_{5}$ \\
\hline$\left(\vartheta_{1}, x, 1\right)$ & $(0.50,0.30)$ & $(0.50,0.20)$ & $(0.80,0.10)$ & $(0.40,0.30)$ & $(0.80,0.10)$ \\
$\left(\vartheta_{2}, x, 1\right)$ & $(0.50,0.20)$ & $(0.60,0.20)$ & $(0.80,0.00)$ & $(0.50,0.30)$ & $(0.40,0.40)$ \\
\hline
\end{tabular}

Hence, it proves that $\left(\gamma_{1}, \chi_{1}, \mathscr{A}\right) \widehat{U}\left(\gamma_{2}, \chi_{2}, \mathscr{B}\right)=\left(\gamma_{2}, \chi_{2}\right.$, $\mathscr{B}) \widehat{U}\left(\gamma_{1}, \chi_{1}, \mathscr{A}\right)$.

Proofs of other parts are similar to part 1.

Definition 17. Restricted fuzzy union of two FBSESs $(\gamma, \chi, \mathscr{A})$ and $\left(\gamma_{1}, \chi_{1}, \mathscr{B}\right)$ over the common universe $\mathcal{U}$ results in a new FBSES $(\gamma, \chi, \mathscr{A}) \widehat{\cup}_{R}\left(\gamma_{1}, \chi_{1}, \mathscr{B}\right)=\left(H_{\cup}^{\prime}, I_{\cap}^{\prime}, C\right)$ over $\mathcal{U}$, where $C=\mathscr{A} \cap \mathscr{B} \neq \varnothing$ and $\forall \alpha \in C$,

$$
\begin{gathered}
H_{\cup}^{\prime}(\alpha)=\max \left(\gamma(\alpha), \gamma_{1}(\alpha)\right), \\
I_{\cap}^{\prime}(\alpha)=\min \left(\chi(\alpha), \chi_{1}(\alpha)\right) .
\end{gathered}
$$

Definition 18. Restricted fuzzy intersection of two FBSESs $(\gamma, \chi, \mathscr{A})$ and $\left(\gamma_{1}, \chi_{1}, \mathscr{B}\right)$ on universe $\mathcal{U}$ results in a new
FBSES $(\gamma, \chi, \mathscr{A}) \widehat{\cap}_{R}\left(\gamma_{1}, \chi_{1}, \mathscr{B}\right)=\left(H_{\cap}^{\prime}, I_{\cup}^{\prime}, C\right)$ over $\mathcal{U}$, where $C=\mathscr{A} \cap \mathscr{B} \neq \varnothing$ and $\forall \alpha \in C$,

$$
\begin{aligned}
H_{\cap}^{\prime}(\alpha) & =\min \left(\gamma(\alpha), \gamma_{1}(\alpha)\right), \\
I_{\cup}^{\prime}(\alpha) & =\max \left(\chi(\alpha), \chi_{1}(\alpha)\right) .
\end{aligned}
$$

Example 9. Consider the FBSESs $(\gamma, \chi, \mathscr{A})$ and $\left(\gamma_{1}, \chi_{1}, \mathscr{B}\right)$ in Example 7. If $C=\mathscr{A} \cap \mathscr{B}$, then by Definition 17 , their restricted union is defined in Table 17.

Similarly by Definition 18 , we have the restricted intersection in Table 18 as $(\gamma, \chi, \mathscr{A}) \widehat{\cap}_{R} \quad\left(\gamma_{1}, \chi_{1}, \mathscr{B}\right)$ $=\left(H_{\cap}^{\prime}, I_{\cup}^{\prime}, C\right)$.

Proposition 5. If $\left(\gamma_{1}, \chi_{1}, \mathscr{A}\right),\left(\gamma_{2}, \chi_{2}, \mathscr{B}\right)$, and $\left(\gamma_{3}, \chi_{3}, \mathscr{C}\right)$ are three FBSESs on $\mathcal{U}$, then 
TABLE 18: Restricted intersection $\left(H_{\cap}^{\prime}, I_{\cup}^{\prime}, C\right)$.

\begin{tabular}{lcccrr}
\hline$\left(H_{\cap}^{\prime}, I_{\cup}^{\prime}, C\right)$ & $u_{1}$ & $u_{2}$ & $u_{3}$ & $u_{4}$ & $u_{5}$ \\
\hline$\left(\vartheta_{1}, x, 1\right)$ & $(0.40,0.40)$ & $(0.50,0.30)$ & $(0.70,0.20)$ & $(0.30,0.40)$ & $(0.70,0.10)$ \\
$\left(\vartheta_{2}, x, 1\right)$ & $(0.50,0.40)$ & $(0.50,0.20)$ & $(0.80,0.10)$ & $(0.50,0.30)$ & $(0.30,0.50)$ \\
\hline
\end{tabular}

(1) $\left(\gamma_{1}, \chi_{1}, \mathscr{A}\right) \widehat{U}_{R}\left(\gamma_{2}, \chi_{2}, \mathscr{B}\right)=\left(\gamma_{2}, \chi_{2}, \mathscr{B}\right) \widehat{U}_{R}$ $\left(\gamma_{1}, \chi_{1}, \mathscr{A}\right)$

(2) $\left(\gamma_{1}, \chi_{1}, \mathscr{A}\right) \widehat{\cap}_{R}\left(\gamma_{2}, \chi_{2}, \mathscr{B}\right)=\left(\gamma_{2}, \chi_{2}, \mathscr{B}\right) \widehat{\cap}_{R}\left(\gamma_{1}, \chi_{1}\right.$, A)

(3) $\left(\gamma_{1}, \chi_{1}, \mathscr{A}\right) \widehat{U}_{R}\left(\left(\gamma_{2}, \chi_{2}, \mathscr{B}\right) \widehat{U}_{R}\left(\gamma_{3}, \chi_{3}, \mathscr{C}\right)\right)=\left(\left(\gamma_{1}, \chi_{1}\right.\right.$, A) $\left.\bar{U}_{R}\left(\gamma_{2}, \chi_{2}, \mathscr{B}\right)\right) \frac{U_{R}}{U_{3}}\left(\gamma_{3}, \mathscr{C}\right)$
(4) $\left(\gamma_{1}, \chi_{1}, \mathscr{A}\right) \widehat{\cap}_{R}\left(\left(\gamma_{2}, \chi_{2}, \mathscr{B}\right) \widehat{\cap}_{R}\left(\gamma_{3}, \chi_{3}, \mathscr{C}\right)\right)=\left(\left(\gamma_{1}, \chi_{1}\right.\right.$, $\left.\mathscr{A}) \widehat{\cap}_{R}\left(\gamma_{2}, \chi_{2}, \mathscr{B}\right)\right) \widehat{\cap}_{R}\left(\gamma_{3}, \chi_{3}, \mathscr{C}\right)$

Proof. (1) For all $\alpha \in \mathscr{A} \cap \mathscr{B}$,

$$
\left(\gamma_{1} \widehat{\cup}_{R} \gamma_{2}\right)(\alpha)=\max \left(\gamma_{1}(\alpha), \gamma_{2}(\alpha)\right)=\max \left(\gamma_{2}(\alpha), \gamma_{1}(\alpha)\right)=\left(\gamma_{2} \widehat{U}_{R} \gamma_{1}\right)(\alpha)
$$

Similarly for all $\alpha \in \mathscr{A} \cap \mathscr{B}$,

$$
\left(\chi_{1} \widehat{\cup}_{R} \chi_{2}\right)(\alpha)=\min \left(\chi_{1}(\alpha), \chi_{2}(\alpha)\right)=\min \left(\chi_{2}(\alpha), \chi_{1}(\alpha)\right)=\left(\chi_{2} \widehat{U}_{R} \chi_{1}\right)(\alpha) .
$$

Hence

$\widehat{\cup}_{R}\left(\gamma_{1}, \chi_{1}, \mathscr{A}\right)$.

Proofs of other parts are similar to part 1.

Proposition 6. If $(\gamma, \chi, \mathscr{A})$ and $\left(\gamma_{1}, \chi_{1}, \mathscr{B}\right)$ are two FBSESs on $\mathcal{U}$, then

(1) $\left((\gamma, \chi, \mathscr{A}) \widehat{U}\left(\gamma_{1}, \chi_{1}, \mathscr{B}\right)\right)^{c}=(\gamma, \chi, \mathscr{A})^{c} \widehat{\cap}\left(\gamma_{1}, \chi_{1}, \mathscr{B}\right)^{c}$

(2) $\left((\gamma, \chi, \mathscr{A}) \widehat{\cap}\left(\gamma_{1}, \chi_{1}, \mathscr{B}\right)\right)^{c}=(\gamma, \chi, \mathscr{A})^{c} \widehat{U}\left(\gamma_{1}, \chi_{1}, \mathscr{B}\right)^{c}$
(3) $\left((\gamma, \chi, \mathscr{A}) \widehat{U}_{R}\left(\gamma_{1}, \chi_{1}, \mathscr{B}\right)\right)^{c}=(\gamma, \chi, \mathscr{A})^{c}$ $\widehat{\cap}_{R}\left(\gamma_{1}, \chi_{1}, \mathscr{B}\right)^{c}$

(4) $\left((\gamma, \chi, \mathscr{A}) \widehat{\cap}_{R}\left(\gamma_{1}, \chi_{1}, \mathscr{B}\right)\right)^{c}=(\gamma, \chi, \mathscr{A})^{c} \widehat{\cup}_{R}$ $\left(\gamma_{1}, \chi_{1}, \mathscr{B}\right)^{c}$

Proof. (1) There are 3 cases for $\alpha \in \mathscr{A} \cup \mathscr{B}$ :

(i) If $\alpha \in \mathscr{A}-\mathscr{B}$, then we have

$$
\left(\gamma \widehat{\cup} \gamma_{1}\right)^{c}(\alpha)=(\gamma)^{c}=\left(\gamma^{c} \widehat{\cap} \gamma_{1}^{c}\right)(\alpha),\left(\chi \widehat{\cup} \chi_{1}\right)^{c}(\alpha)=(\chi)^{c}=\left(\chi^{c} \widehat{\cap} \chi_{1}^{c}\right)(\alpha) .
$$

(ii) If $\alpha \in \mathscr{B}-\mathscr{A}$, then we have

$$
\left(\gamma \widehat{\cup} \gamma_{1}\right)^{c}(\alpha)=\left(\gamma_{1}\right)^{c}=\left(\gamma^{c} \widehat{\cap} \gamma_{1}^{c}\right)(\alpha),\left(\chi \widehat{\cup} \chi_{1}\right)^{c}(\alpha)=\left(\chi_{1}\right)^{c}=\left(\chi^{c} \widehat{\cap} \chi_{1}^{c}\right)(\alpha) .
$$

(iii) If $\alpha \in \mathscr{A} \cap \mathscr{B}$, then we have

$$
\begin{aligned}
& \left(\gamma \hat{\cup} \gamma_{1}\right)^{c}(\alpha)=\left(\max \left(\gamma(\alpha), \gamma_{1}(\alpha)\right)\right)^{c}=\min \left(\gamma^{c}(\alpha), \gamma_{1}^{c}(\alpha)\right)=\left(\gamma^{c} \hat{\cup} \gamma_{1}^{c}\right)(\alpha), \\
& \left(\chi \hat{\cup} \chi_{1}\right)^{c}(\alpha)=\left(\min \left(\chi(\alpha), \chi_{1}(\alpha)\right)\right)^{c}=\max \left(\chi^{c}(\alpha), \chi_{1}^{c}(\alpha)\right)=\left(\chi^{c} \hat{\cup} \chi_{1}^{c}\right)(\alpha) .
\end{aligned}
$$

In all three cases, it is obvious that

$$
\left((\gamma, \chi, \mathscr{A}) \widehat{\cup}\left(\gamma_{1}, \chi_{1}, \mathscr{B}\right)\right)^{c}=(\gamma, \chi, \mathscr{A})^{c} \widehat{\cap}\left(\gamma_{1}, \chi_{1}, \mathscr{B}\right)^{c} .
$$

Similarly, we can prove the remaining parts.
Proposition 7. If $(\gamma, \chi, \mathscr{A})$ and $\left(\gamma_{1}, \chi_{1}, \mathscr{A}\right)$ are two FBSESs over $\mathcal{U}$, then

(1) $(\gamma, \chi, \mathscr{A}) \widehat{\mathrm{U}}\left(\gamma_{1}, \chi_{1}, \mathscr{A}\right)=(\gamma, \chi, \mathscr{A}) \widehat{\mathrm{U}}_{R}\left(\gamma_{1}, \chi_{1}, \mathscr{A}\right)$

(2) $(\gamma, \chi, \mathscr{A}) \widehat{\cap}\left(\gamma_{1}, \chi_{1}, \mathscr{A}\right)=(\gamma, \chi, \mathscr{A}) \widehat{\cap}_{R}\left(\gamma_{1}, \chi_{1}, \mathscr{A}\right)$

(3) $(\gamma, \chi, \mathscr{A}) \widehat{\mathrm{U}}(\gamma, \chi, \mathscr{A})=(\gamma, \chi, \mathscr{A})$ 


$$
\begin{aligned}
& \left(\gamma \hat{\cup} \gamma_{1}\right)(\alpha)=\max \left(\gamma(\alpha), \gamma_{1}(\alpha)\right)=\left(\gamma \hat{\cup}_{R} \gamma_{1}\right)(\alpha), \\
& \left(\chi \widehat{\cup} \chi_{1}\right)(\alpha)=\min \left(\chi(\alpha), \chi_{1}(\alpha)\right)=\left(\chi \widehat{\cup}_{R} \chi_{1}\right)(\alpha) .
\end{aligned}
$$

Hence, $(\gamma, \chi, \mathscr{A}) \widehat{U}\left(\gamma_{1}, \chi_{1}, \mathscr{A}\right)=(\gamma, \chi, \mathscr{A}) \widehat{\mathrm{U}}_{R}\left(\gamma_{1}, \chi_{1}, \mathscr{A}\right)$. The remaining parts can be proved in a similar fashion.

Proposition 8. If $(\Phi, \mathfrak{U}, \mathscr{A})$ is a null FBSES, $(\mathfrak{U}, \Phi, \mathscr{A})$, an absolute FBSES, and $(\gamma, \chi, \mathscr{A})$ is any other FBSES on $\mathcal{U}$, then

(1) $(\gamma, \chi, \mathscr{A}) \widehat{\cup}(\Phi, \mathfrak{U}, \mathscr{A})=(\gamma, \chi, \mathscr{A})$

(2) $(\gamma, \chi, \mathscr{A}) \widehat{\cap}(\Phi, \mathfrak{U}, \mathscr{A})=(\Phi, \mathfrak{U}, \mathscr{A})$

(3) $(\gamma, \chi, \mathscr{A}) \widehat{\cup}(\mathfrak{U}, \Phi, \mathscr{A})=(\mathfrak{U}, \Phi, \mathscr{A})$

(4) $(\gamma, \chi, \mathscr{A}) \widehat{\cap}(\mathcal{U}, \Phi, \mathscr{A})=(\gamma, \chi, \mathscr{A})$

\section{Proof}

(1) For all $\alpha \in \mathscr{A} \cup \mathscr{A}=\mathscr{A} \cap \mathscr{A}=\mathscr{A}$, we have

$(\gamma \widehat{\cup} \Phi)(\alpha)=\max (\gamma(\alpha), \Phi(\alpha))=\max (\gamma(\alpha), 0)=\gamma(\alpha)$,

and for all $\alpha \in \mathscr{A} \cup \mathscr{A}=\mathscr{A} \cap \mathscr{A}=\mathscr{A}$,

$(\chi \widehat{\cup} \mathfrak{U})(\alpha)=\min (\chi(\alpha), \mathfrak{U}(\alpha))=\min (\chi(\alpha), 1)=\chi(\alpha)$.

Hence, $(\gamma, \chi, \mathscr{A}) \widehat{\cup}(\Phi, \mathcal{U}, \mathscr{A})=(\gamma, \chi, \mathscr{A})$.

(2) For all $\alpha \in \mathscr{A} \cup \mathscr{A}=\mathscr{A}=\mathscr{A} \cap \mathscr{A}$, we have

$(\gamma \widehat{\cap} \Phi)(\alpha)=\min (\gamma(\alpha), \Phi(\alpha))=\min (\gamma(\alpha), 0)=0=\Phi(\alpha)$,

and for all $\alpha \in \mathscr{A} \cup \mathscr{A}=\mathscr{A}=\mathscr{A} \cap \mathscr{A}$, $(\chi \widehat{\cap} \mathfrak{U})(\alpha)=\max (\chi(\alpha), \mathfrak{U}(\alpha))=\max (\chi(\alpha), 1)=1=\mathfrak{U}(\alpha)$.

Hence, $(\gamma, \chi, \mathscr{A}) \widehat{\cap}(\Phi, \mathfrak{U}, \mathscr{A})=(\Phi, \mathfrak{U}, \mathscr{A})$.

The remaining parts can be easily verified with similar arguments.

Proposition 9. Let $\left(\gamma_{1}, \chi_{1}, \mathscr{A}\right),\left(\gamma_{2}, \chi_{2}, \mathscr{B}\right)$, and $\left(\gamma_{3}, \chi_{3}, \mathscr{C}\right)$ be any three FBSESs on $\mathcal{U}$, then

(1) $\left(\gamma_{1}, \chi_{1}, \mathscr{A}\right) \widehat{\cap}_{R}\left(\left(\gamma_{2}, \chi_{2}, \mathscr{B}\right) \widehat{U}\left(\gamma_{3}, \chi_{3}, \mathscr{C}\right)\right)=\left(\left(\gamma_{1}, \chi_{1}\right.\right.$, $\left.\mathscr{A}) \widehat{\cap}_{R}\left(\gamma_{2}, \chi_{2}, \mathscr{B}\right)\right) \cup\left(\left(\gamma_{1}, \chi_{1}, \mathscr{A}\right) \widehat{\cap}_{R}\left(\gamma_{3}, \chi_{3}, \mathscr{C}\right)\right)$

(2) $\left(\gamma_{1}, \chi_{1}, \mathscr{A}\right) \widehat{U}_{R}\left(\left(\gamma_{2}, \chi_{2}, \mathscr{B}\right) \quad \widehat{\cap}\left(\gamma_{3}, \chi_{3}, \mathscr{C}\right)\right)=\left(\left(\gamma_{1}\right.\right.$, $\left.\left.\chi_{1}, \mathscr{A}\right) \widehat{U}_{R}\left(\gamma_{2}, \chi_{2}, \mathscr{B}\right)\right) \widehat{\cap}\left(\left(\gamma_{1}, \chi_{1}, \mathscr{A}\right) \widehat{U}_{R}\left(\gamma_{3}, \chi_{3}, \mathscr{C}\right)\right)$

(3) $\left(\gamma_{1}, \chi_{1}, \mathscr{A}\right) \widehat{\cap}\left(\left(\gamma_{2}, \chi_{2}, \mathscr{B}\right) \widehat{U}_{R}\left(\gamma_{3}, \chi_{3}, \mathscr{C}\right)\right)=\left(\left(\gamma_{1}, \chi_{1}\right.\right.$, $\left.\mathscr{A}) \hat{\cap}\left(\gamma_{2}, \chi_{2}, \mathscr{B}\right)\right) \widehat{U}_{R}\left(\left(\gamma_{1}, \chi_{1}, \mathscr{A}\right) \hat{\cap}\left(\gamma_{3}, \chi_{3}, \mathscr{C}\right)\right)$

(4) $\left(\gamma_{1}, \chi_{1}, \mathscr{A}\right) \widehat{U}\left(\left(\gamma_{2}, \chi_{2}, \mathscr{B}\right) \widehat{\cap}_{R} \quad\left(\gamma_{3}, \chi_{3}, \mathscr{C}\right)\right)=\left(\left(\gamma_{1}\right.\right.$, $\left.\left.\chi_{1}, \mathscr{A}\right) \widehat{U}\left(\gamma_{2}, \chi_{2}, \mathscr{B}\right)\right) \widehat{\cap}_{R}\left(\left(\gamma_{1}, \chi_{1}, \mathscr{A}\right) \widehat{U}\left(\gamma_{3}, \chi_{3}, \mathscr{C}\right)\right)$

(5) $\left(\gamma_{1}, \chi_{1}, \mathscr{A}\right) \widehat{\cap}_{R}\left(\left(\gamma_{2}, \chi_{2}, \mathscr{B}\right) \widehat{\cup}_{R}\left(\gamma_{3}, \chi_{3}, \mathscr{C}\right)\right)=\left(\left(\gamma_{1}, \chi_{1}\right.\right.$, $\left.\mathscr{A}) \widehat{\cap}_{R}\left(\gamma_{2}, \chi_{2}, \mathscr{B}\right)\right) \widehat{U}_{R}\left(\left(\gamma_{1}, \chi_{1}, \mathscr{A}\right) \widehat{\cap}_{R}\left(\gamma_{3}, \chi_{3}, \mathscr{C}\right)\right)$

(6) $\left(\gamma_{1}, \chi_{1}, \mathscr{A}\right) \widehat{\cup}_{R}\left(\left(\gamma_{2}, \chi_{2}, \mathscr{B}\right) \widehat{\cap}_{R}\left(\gamma_{3}, \chi_{3}, \mathscr{C}\right)\right)=\left(\left(\gamma_{1}, \chi_{1}\right.\right.$, $\left.\mathscr{A}) \widehat{U}_{R}\left(\gamma_{2}, \chi_{2}, \mathscr{B}\right)\right) \widehat{\cap}_{R}\left(\left(\gamma_{1}, \chi_{1}, \mathscr{A}\right) \widehat{U}_{R}\left(\gamma_{3}, \chi_{3}, \mathscr{C}\right)\right)$

Proof

(1) Consider $\alpha \in \mathscr{A} \cap(\mathscr{B} \cup C)$. Then, three possibilities exist:

(i) If $\alpha \in \mathscr{A} \cap(\mathscr{B}-C)$, then

$$
\begin{aligned}
\gamma_{1}(\alpha) \widehat{\Pi}_{R}\left(\gamma_{2} \widehat{U} \gamma_{3}\right)(\alpha) & =\gamma_{1}(\alpha) \widehat{\Pi}_{R} \gamma_{2}(\alpha)=\min \left(\gamma_{1}(\alpha), \gamma_{2}(\alpha)\right), \\
\chi_{1}(\alpha) \hat{\cap}_{R}\left(\chi_{2} \hat{U} \chi_{3}\right)(\alpha) & =\chi_{1}(\alpha) \widehat{\cap}_{R} \chi_{2}(\alpha)=\max \left(\chi_{1}(\alpha), \chi_{2}(\alpha)\right), \\
\left(\gamma_{1} \widehat{\cap}_{R} \gamma_{2}\right)(\alpha) \widehat{U}\left(\gamma_{1} \widehat{\cap}_{R} \gamma_{3}\right)(\alpha) & =\max \left(\left(\gamma_{1} \widehat{\cap}_{R} \gamma_{2}\right)(\alpha), \varnothing\right)=\min \left(\gamma_{1}(\alpha), \gamma_{2}(\alpha)\right), \\
\left(\chi_{1} \widehat{\cap}_{R} \chi_{2}\right)(\alpha) \widehat{U}\left(\chi_{1} \widehat{\cap}_{R} \chi_{3}\right)(\alpha) & =\min \left(\left(\chi_{1} \widehat{\cap}_{R} \chi_{2}\right)(\alpha), \mathcal{U}\right)=\max \left(\chi_{1}(\alpha), \chi_{2}(\alpha)\right) .
\end{aligned}
$$

(ii) If $\alpha \in \mathscr{A} \cap(C-\mathscr{B})$, then

$$
\begin{aligned}
\gamma_{1}(\alpha) \widehat{\cap}_{R}\left(\gamma_{2} \widehat{U} \gamma_{3}\right)(\alpha) & =\gamma_{1}(\alpha) \widehat{\cap}_{R} \gamma_{3}(\alpha)=\min \left(\gamma_{1}(\alpha), \gamma_{3}(\alpha)\right), \\
\chi_{1}(\alpha) \widehat{\cap}_{R}\left(\chi_{2} \widehat{U} \chi_{3}\right)(\alpha) & =\chi_{1}(\alpha) \widehat{\cap}_{R} \chi_{3}(\alpha)=\max \left(\chi_{1}(\alpha), \chi_{3}(\alpha)\right), \\
\left(\gamma_{1} \widehat{\cap}_{R} \gamma_{2}\right)(\alpha) \widehat{U}\left(\gamma_{1} \widehat{\cap}_{R} \gamma_{3}\right)(\alpha) & =\max \left(\varnothing,\left(\gamma_{1} \widehat{\cap}_{R} \gamma_{3}\right)(\alpha)\right)=\min \left(\gamma_{1}(\alpha), \gamma_{3}(\alpha)\right), \\
\left(\chi_{1} \widehat{\cap}_{R} \chi_{2}\right)(\alpha) \widehat{U}\left(\chi_{1} \widehat{\cap}_{R} \chi_{3}\right)(\alpha) & =\min \left(\mathcal{U},\left(\chi_{1} \widehat{\cap}_{R} \chi_{3}\right)(\alpha)\right)=\max \left(\chi_{1}(\alpha), \chi_{3}(\alpha)\right) .
\end{aligned}
$$


(iii) If $\alpha \in \mathscr{A} \cap(\mathscr{B} \cap C)$, then

$$
\begin{aligned}
\gamma_{1}(\alpha) \widehat{\cap}_{R}\left(\gamma_{2} \widehat{U} \gamma_{3}\right)(\alpha) & =\gamma_{1}(\alpha) \widehat{\cap}_{R} \max \left(\gamma_{2}(\alpha), \gamma_{3}(\alpha)\right)=\min \left(\gamma_{1}(\alpha), \max \left(\gamma_{2}(\alpha), \gamma_{3}(\alpha)\right)\right), \\
\chi_{1}(\alpha) \widehat{\Pi}_{R}\left(\chi_{2} \widehat{U} \chi_{3}\right)(\alpha) & =\chi_{1}(\alpha) \widehat{\cap}_{R} \min \left(\chi_{2}(\alpha), \chi_{3}(\alpha)\right)=\max \left(\chi_{1}(\alpha), \min \left(\chi_{2}(\alpha), \chi_{3}(\alpha)\right)\right), \\
\left(\gamma_{1} \widehat{\cap}_{R} \gamma_{2}\right)(\alpha) \widehat{U}\left(\gamma_{1} \widehat{\cap}_{R} \gamma_{3}\right)(\alpha) & =\max \left(\left(\gamma_{1} \widehat{\cap}_{R} \gamma_{2}\right)(\alpha),\left(\gamma_{1} \widehat{\cap}_{R} \gamma_{3}\right)(\alpha)\right) \\
& =\max \left(\min \left(\gamma_{1}(\alpha), \gamma_{2}(\alpha)\right), \min \left(\gamma_{1}(\alpha) \cap \gamma_{3}(\alpha)\right)\right) \\
& =\min \left(\gamma_{1}(\alpha), \max \left(\gamma_{2}(\alpha), \gamma_{3}(\alpha)\right)\right),\left(\chi_{1} \widehat{\cap}_{R} \chi_{2}\right)(\alpha) \widehat{U}\left(\chi_{1} \widehat{\cap}_{R} \chi_{3}\right)(\alpha) \\
& =\min \left(\left(\chi_{1} \widehat{\cap}_{R} \chi_{2}\right)(\alpha),\left(\chi_{1} \widehat{\cap}_{R} \chi_{3}\right)(\alpha)\right) \\
= & \min \left(\max \left(\chi_{1}(\alpha), \chi_{2}(\alpha)\right), \max \left(\chi_{1}(\alpha), \chi_{3}(\alpha)\right)\right) \\
= & \max \left(\chi_{1}(\alpha), \min \left(\chi_{2}(\alpha), \chi_{3}(\alpha)\right)\right) .
\end{aligned}
$$

Hence, we prove that in all 3 possibilities, we have $\left(\gamma_{1}, \chi_{1}, \mathscr{A}\right) \widehat{\cap}_{R}\left(\left(\gamma_{2}, \chi_{2}, \mathscr{B}\right) \widehat{U} \quad\left(\gamma_{3}, \chi_{3}, C\right)\right)=\left(\left(\gamma_{1}, \chi_{1}, \mathscr{A}\right) \widehat{\Pi}_{R}\right.$ $\left.\left(\gamma_{2}, \chi_{2}, \mathscr{B}\right)\right) \hat{U}\left(\left(\gamma_{1}, \chi_{1}, \mathscr{A}\right) \widehat{\cap}_{R}\left(\gamma_{3}, \chi_{3}, C\right)\right)$.

Similarly, we can prove the remaining parts.

\section{Application of FBSESs in a MAGDM Problem}

In this section, we will use the proposed FBSES model to deal with the uncertainties in a real-life problem.

Definition 19. For agree and disagree FBSES tables with entries $a_{i j}=\left(\gamma_{i j}, \chi_{i j}\right)$, the focus agree ( $f$-agree) FBSES and focus disagree ( $f$-disagree) FBSES tables consist of $i$-th rows and $j$-th columns with entries $f_{i j}$ defined as

$$
f_{i j}=\gamma_{i j}-\chi_{i j}
$$

Definition 20. The $f$-agree score $\stackrel{\circ}{\xi}_{j}$ of an object $u_{i} \in \mathscr{U}$ is defined as

$$
\stackrel{\circ}{\xi}=\sum_{i} f_{i j} \text {, }
$$

where $f_{i j}$ is the $i j$-th entry of the focus agree FBSES table.

Definition 21. The $f$-disagree score $\dot{\eta}_{j}$ of an object $u_{i} \in \mathcal{U}$ is defined as

$$
\stackrel{\circ}{\eta}=\sum_{i} f_{i j}
$$

where $f_{i j}$ is the $i j$-th entry of the focus disagree FBSES table.

Example 10. Ranking effectiveness of tests for spread analysis of COVID-19.

COVID-19 is a highly contagious disease caused by SARS-CoV-2 (severe acute respiratory syndrome coronavirus 2). After being first time informed to the WHO (World Health Organization) on December 31, 2019, when several cases of an unknown pneumonia sort of disease were reported in the Wuhan city of China, this viral disease has been the most important concern for the whole humanity. On March 11, 2020, the WHO Director General officially categorized this COVID-19 outbreak as a pandemic on the basis of rapid increase in cases extending outside China all over the world. The outbreak has been declared to be a world health emergency by the WHO.

This novel and highly lethal disease has proved to spread successfully in all weather conditions and all health standards (though a bit slower in certain situations). People already suffering with chronic diseases such as diabetes, cardio problems, and respiratory diseases are proved to be more vulnerable to get the virus. This virus hits all ages and genders leaving no one safe to walk free. Children not very vulnerable to the disease are otherwise highly affected by the socioeconomic changes and the fear initiated by the outbreak. Strict lockdowns, closure of educational institutions, lack of supplies, etc., caused by the outbreak are also considerable factors ruining the economies and societies.

This disease has proved to be a game changer since its outbreak changing economies, restricting travels, isolating countries, increasing casualties massively, and making major demographic changes. The virus causing COVID-19 is mutating at a high rate and is, therefore, highly unpredictable. According to the recent updates by the WHO as of March 20, 2021, there have been 121,969,223 confirmed cases globally of COVID-19, including 2,694,094 deaths reported, and the numbers are still increasing. Since this is a novel disease and is mutating, we do not have a medication for the treatment of this disease. Different countries and institutions claimed for different medicines to be helpful in the treatment of COVID-19, but later proved to be wrong. Different countries are developing and have developed vaccines to save people from getting infected, but the availability of vaccination to each and every person will take a lot of time. Moreover, these vaccines might not work with the virus's new strands and have a success rate up to $80 \%$ to $90 \%$ only. It is, therefore, very necessary to keep track of the spread effectively, so that it may be restricted from moving to less-affected or not-affected areas.

Currently, different types of tests are available for COVID19 playing a vital role in analyzing the spread of this disease and helping the decision-makers and governments in making policies, respectively. Let $\mathcal{U}=\left\{u_{1}, u_{2}, u_{3}, u_{4}\right\}$ be the set of available tests for COVID-19, where 
(i) $u_{1}$ : rRT-PCR (real-time reverse transcription polymerase chain reaction) is a diagnosis test for COVID-19 with high specificity and sensitivity. The test is conducted using nasal or mouth swabs.

(ii) $u_{2}$ : CBNAAT (cartridge-based nucleic acid amplification test) is a diagnosis test formerly used in TB diagnosis, now used in some countries for COVID19 diagnosis. It is faster than RT-PCR.

(iii) $u_{3}$ : Antigen tests target the spike proteins of coronavirus in order to detect it as a foreign material. Samples are taken by nasal swabs.

(iv) $u_{4}$ : Antibody tests also called serological tests for COVID-19 detect antibodies created in a body against COVID-19. This test detects prior and ongoing infections by detecting $\operatorname{IgG}$ and $\operatorname{IgM}$ antibodies. Blood sample is used in this test.

Consider $E=\{x, y\}$ to be the set of 2 agencies analyzing the above-discussed COVID-19 diagnosis tests individually. The agencies consider the set $\mathscr{V}=\left\{\vartheta_{1}, \vartheta_{2}, \ldots, \vartheta_{10}\right\}$ as the set of favorable characteristics of a good test, such that

(i) $\vartheta_{1}$ : sensitivity $=($ true positives/true positives + false negatives)

(ii) $\vartheta_{2}: \quad$ specificity $=($ true negatives/true negatives + false positives)

(iii) $\vartheta_{3}$ : positive predictive value $(\mathrm{PPV})=($ true positives/true positives + false positives) at $5 \%$ prevalence

(iv) $\vartheta_{4}: \mathrm{PPV}$ at $10 \%$ prevalence

(v) $\vartheta_{5}: \mathrm{PPV}$ at $15 \%$ prevalence

(vi) $\vartheta_{6}$ : negative predictive value $(\mathrm{NPV})=($ true negatives/true negatives + false negatives) at $5 \%$ prevalence

(vii) $\vartheta_{7}: \mathrm{NPV}$ at $10 \%$ prevalence

(viii) $\vartheta_{8}: \mathrm{NPV}$ at $15 \%$ prevalence

(ix) $\vartheta_{9}$ : low test load (meaning minimum time, cost, training, and equipment)

(x) $\vartheta_{10}$ : independent (independent of age, disease severity, and no reconfirmation by further tests)

However, the set $\mathscr{V}=\left\{\vartheta_{1}, \vartheta_{2}, \ldots, \vartheta_{10}\right\}$ is considered as the set of unfavorable characteristics, such that

(i) $\vartheta_{1}$ : false-negative rate $(\mathrm{FNR})=($ false negatives $/$ true positives + false negatives)

(ii) $\vartheta_{2}$ : false-positive rate $(\mathrm{FPR})=($ false positives $/$ true negatives + false positives)

(iii) $\vartheta_{3}$ : false discovery rate $($ FDR $)=($ false positives/true positives + false positives) at $5 \%$ prevalence

(iv) $\vartheta_{4}:$ FDR at $10 \%$ prevalence

(v) $\vartheta_{5}:$ FDR at $15 \%$ prevalence

(vi) $\vartheta_{6}$ : false omission rate $(\mathrm{FOR})=($ false negatives $/$ true negatives + false negatives) at $5 \%$ prevalence

(vii) $\vartheta_{7}:$ FOR at $10 \%$ prevalence
TABle 19: Agree FBSES $(\gamma, \chi, X)_{1}$.

\begin{tabular}{lcccc}
\hline$(\gamma, \chi, X)_{1}$ & $u_{1}$ & $u_{2}$ & $u_{3}$ & $u_{4}$ \\
\hline$\left(\vartheta_{1}, x\right)$ & $(0.90,0.10)$ & $(0.80,0.20)$ & $(0.70,0.30)$ & $(0.80,0.20)$ \\
$\left(\vartheta_{1}, y\right)$ & $(0.93,0.07)$ & $(0.75,0.25)$ & $(0.55,0.45)$ & $(0.87,0.13)$ \\
$\left(\vartheta_{2}, x\right)$ & $(0.95,0.05)$ & $(0.92,0.08)$ & $(0.85,0.15)$ & $(0.80,0.20)$ \\
$\left(\vartheta_{2}, y\right)$ & $(0.97,0.03)$ & $(0.90,0.10)$ & $(0.90,0.10)$ & $(0.98,0.02)$ \\
$\left(\vartheta_{3}, x\right)$ & $(0.48,0.51)$ & $(0.34,0.65)$ & $(0.20,0.80)$ & $(0.17,0.83)$ \\
$\left(\vartheta_{3}, y\right)$ & $(0.62,0.38)$ & $(0.28,0.72)$ & $(0.22,0.77)$ & $(0.69,0.30)$ \\
$\left(\vartheta_{4}, x\right)$ & $(0.66,0.33)$ & $(0.53,0.47)$ & $(0.34,0.66)$ & $(0.31,0.69)$ \\
$\left(\vartheta_{4}, y\right)$ & $(0.78,0.22)$ & $(0.45,0.54)$ & $(0.38,0.62)$ & $(0.83,0.17)$ \\
$\left(\vartheta_{5}, x\right)$ & $(0.76,0.23)$ & $(0.64,0.36)$ & $(0.45,0.55)$ & $(0.41,0.59)$ \\
$\left(\vartheta_{5}, y\right)$ & $(0.84,0.15)$ & $(0.57,0.43)$ & $(0.49,0.51)$ & $(0.88,0.11)$ \\
$\left(\vartheta_{6}, x\right)$ & $(0.99,0.00)$ & $(0.99,0.01)$ & $(0.98,0.02)$ & $(0.99,0.01)$ \\
$\left(\vartheta_{6}, y\right)$ & $(0.99,0.00)$ & $(0.98,0.01)$ & $(0.97,0.02)$ & $(0.99,0.01)$ \\
$\left(\vartheta_{7}, x\right)$ & $(0.98,0.01)$ & $(0.97,0.02)$ & $(0.96,0.04)$ & $(0.97,0.03)$ \\
$\left(\vartheta_{7}, y\right)$ & $(0.99,0.01)$ & $(0.97,0.03)$ & $(0.95,0.05)$ & $(0.98,0.01)$ \\
$\left(\vartheta_{8}, x\right)$ & $(0.98,0.02)$ & $(0.96,0.03)$ & $(0.94,0.06)$ & $(0.96,0.04)$ \\
$\left(\vartheta_{8}, y\right)$ & $(0.99,0.01)$ & $(0.95,0.04)$ & $(0.92,0.08)$ & $(0.98,0.02)$ \\
$\left(\vartheta_{9}, x\right)$ & $(0.10,0.80)$ & $(0.60,0.30)$ & $(0.75,0.10)$ & $(0.50,0.50)$ \\
$\left(\vartheta_{9}, y\right)$ & $(0.20,0.70)$ & $(0.80,0.15)$ & $(0.65,0.15)$ & $(0.50,0.45)$ \\
$\left(\vartheta_{10}, x\right)$ & $(0.95,0.00)$ & $(0.80,0.15)$ & $(0.60,0.30)$ & $(0.50,0.50)$ \\
$\left(\vartheta_{10}, y\right)$ & $(0.90,0.05)$ & $(0.75,0.20)$ & $(0.50,0.40)$ & $(0.50,0.50)$ \\
\hline
\end{tabular}

Table 20: Disagree FBSES $(\gamma, \chi, X)_{0}$.

\begin{tabular}{lcccc}
\hline$(\gamma, \chi, X)_{0}$ & $u_{1}$ & $u_{2}$ & $u_{3}$ & $u_{4}$ \\
\hline$\left(\vartheta_{1}, x\right)$ & $(0.10,0.85)$ & $(0.10,0.80)$ & $(0.20,0.70)$ & $(0.15,0.75)$ \\
$\left(\vartheta_{1}, y\right)$ & $(0.05,0.90)$ & $(0.20,0.70)$ & $(0.40,0.50)$ & $(0.10,0.85)$ \\
$\left(\vartheta_{2}, x\right)$ & $(0.02,0.95)$ & $(0.04,0.90)$ & $(0.10,0.85)$ & $(0.10,0.85)$ \\
$\left(\vartheta_{2}, y\right)$ & $(0.00,0.95)$ & $(0.05,0.90)$ & $(0.10,0.80)$ & $(0.00,0.96)$ \\
$\left(\vartheta_{3}, x\right)$ & $(0.50,0.45)$ & $(0.60,0.35)$ & $(0.80,0.20)$ & $(0.80,0.15)$ \\
$\left(\vartheta_{3}, y\right)$ & $(0.35,0.60)$ & $(0.70,0.20)$ & $(0.75,0.15)$ & $(0.30,0.65)$ \\
$\left(\vartheta_{4}, x\right)$ & $(0.30,0.65)$ & $(0.45,0.50)$ & $(0.65,0.35)$ & $(0.60,0.30)$ \\
$\left(\vartheta_{4}, y\right)$ & $(0.20,0.75)$ & $(0.50,0.40)$ & $(0.60,0.35)$ & $(0.15,0.80)$ \\
$\left(\vartheta_{5}, x\right)$ & $(0.20,0.70)$ & $(0.35,0.60)$ & $(0.50,0.40)$ & $(0.55,0.40)$ \\
$\left(\vartheta_{5}, y\right)$ & $(0.10,0.85)$ & $(0.40,0.55)$ & $(0.50,0.45)$ & $(0.10,0.85)$ \\
$\left(\vartheta_{6}, x\right)$ & $(0.01,0.99)$ & $(0.01,0.95)$ & $(0.02,0.95)$ & $(0.01,0.95)$ \\
$\left(\vartheta_{6}, y\right)$ & $(0.01,0.99)$ & $(0.02,0.95)$ & $(0.03,0.94)$ & $(0.01,0.95)$ \\
$\left(\vartheta_{7}, x\right)$ & $(0.02,0.95)$ & $(0.03,0.94)$ & $(0.04,0.90)$ & $(0.03,0.94)$ \\
$\left(\vartheta_{7}, y\right)$ & $(0.01,0.95)$ & $(0.03,0.94)$ & $(0.05,0.90)$ & $(0.02,0.95)$ \\
$\left(\vartheta_{8}, x\right)$ & $(0.02,0.95)$ & $(0.04,0.95)$ & $(0.06,0.90)$ & $(0.04,0.90)$ \\
$\left(\vartheta_{8}, y\right)$ & $(0.01,0.95)$ & $(0.05,0.90)$ & $(0.08,0.90)$ & $(0.02,0.95)$ \\
$\left(\vartheta_{9}, x\right)$ & $(0.90,0.10)$ & $(0.30,0.65)$ & $(0.15,0.80)$ & $(0.50,0.45)$ \\
$\left(\vartheta_{9}, y\right)$ & $(0.75,0.20)$ & $(0.15,0.80)$ & $(0.30,0.60)$ & $(0.45,0.50)$ \\
$\left(\vartheta_{10}, x\right)$ & $(0.05,0.90)$ & $(0.20,0.80)$ & $(0.35,0.60)$ & $(0.50,0.45)$ \\
$\left(\vartheta_{10}, y\right)$ & $(0.10,0.90)$ & $(0.20,0.75)$ & $(0.40,0.50)$ & $(0.50,0.45)$ \\
\hline
\end{tabular}

(viii) $\vartheta_{8}$ : FOR at $15 \%$ prevalence

(ix) $\vartheta_{9}$ : high test load (meaning maximum time, cost, training, and equipment)

(x) $\vartheta_{10}$ : dependent (dependent on age, disease severity, and need confirmation by further tests)

For more clarification about the above-mentioned formulas and tests one can visit the following link: https://en. wikipedia.org/wiki/Sensitivity_and_specificity.

Note that PPV, NPV, FDR, and FOR are prevalencedependent factors and therefore are calculated at three different levels in order to analyze the tests in maximum 
(1) Input the FBSES $(\gamma, \chi, X)$.

(2) Find the agree and disagree FBSES tables with entries $a_{i j}=\left(\gamma_{i j}, \chi_{i j}\right)$.

(3) Find the focus agree and focus disagree tables with entries $f_{i j}=\gamma_{i j}-\chi_{i j}$.

(4) Determine the $f$-agree score $\stackrel{\xi}{\xi} \in D_{1}: \grave{\xi}_{j}=\sum_{i} f_{i j}$ as the last row in the focus agree FBSES table.

(5) Determine the $f$-disagree score $i \dot{\eta} \in D_{0}: \grave{\eta}_{j}=\sum_{i} f_{i j}$ as the last row in the focus disagree FBSES table.

(6) Find the final score $\lambda_{j}=\xi_{j}-\grave{\eta}_{j}$.

(7) Find $k$ for which $\grave{\lambda}_{k}=\max \left(\grave{\lambda}_{j}\right)$.

(8) Rank $u_{k}$ for ascending values of $k$.

Algorithm 1: Ranking of tests based on FBSES.

TABLE 21: $f$-Agree FBSES.

\begin{tabular}{lcccc}
\hline $\mathcal{U}$ & $u_{1}$ & $u_{2}$ & $u_{3}$ & $u_{4}$ \\
\hline$\left(\vartheta_{1}, x\right)$ & 0.80 & 0.60 & 0.40 & 0.60 \\
$\left(\vartheta_{1}, y\right)$ & 0.86 & 0.50 & 0.10 & 0.74 \\
$\left(\vartheta_{2}, x\right)$ & 0.90 & 0.84 & 0.70 & 0.60 \\
$\left(\vartheta_{2}, y\right)$ & 0.94 & 0.80 & 0.80 & 0.96 \\
$\left(\vartheta_{3}, x\right)$ & -0.03 & -0.31 & -0.60 & -0.66 \\
$\left(\vartheta_{3}, y\right)$ & 0.24 & -0.44 & -0.55 & 0.39 \\
$\left(\vartheta_{4}, x\right)$ & 0.33 & 0.06 & -0.32 & -0.38 \\
$\left(\vartheta_{4}, y\right)$ & 0.56 & -0.09 & -0.24 & 0.66 \\
$\left(\vartheta_{5}, x\right)$ & 0.53 & 0.28 & -0.10 & -0.18 \\
$\left(\vartheta_{5}, y\right)$ & 0.69 & 0.14 & -0.02 & 0.77 \\
$\left(\vartheta_{6}, x\right)$ & 0.99 & 0.98 & 0.96 & 0.98 \\
$\left(\vartheta_{6}, y\right)$ & 0.99 & 0.97 & 0.95 & 0.98 \\
$\left(\vartheta_{7}, x\right)$ & 0.97 & 0.95 & 0.92 & 0.94 \\
$\left(\vartheta_{7}, y\right)$ & 0.98 & 0.94 & 0.90 & 0.97 \\
$\left(\vartheta_{8}, x\right)$ & 0.96 & 0.93 & 0.88 & 0.92 \\
$\left(\vartheta_{8}, y\right)$ & 0.98 & 0.91 & 0.84 & 0.96 \\
$\left(\vartheta_{9}, x\right)$ & -0.70 & 0.30 & 0.65 & 0.00 \\
$\left(\vartheta_{9}, y\right)$ & -0.50 & 0.65 & 0.50 & 0.05 \\
$\left(\vartheta_{10}, x\right)$ & 0.95 & 0.65 & 0.30 & 0.00 \\
$\left(\vartheta_{10}, y\right)$ & 0.85 & 0.55 & 0.10 & 0.00 \\
\hline$\xi$ & 12.29 & 10.21 & 7.17 & 9.30 \\
\hline \multirow{9}{*}{} & & & &
\end{tabular}

TABLE 22: $f$-Disagree FBSES.

\begin{tabular}{lcccc}
\hline $\mathcal{U}$ & $u_{1}$ & $u_{2}$ & $u_{3}$ & $u_{4}$ \\
\hline$\left(\vartheta_{1}, x\right)$ & -0.75 & -0.70 & -0.50 & -0.60 \\
$\left(\vartheta_{1}, y\right)$ & -0.85 & -0.50 & -0.10 & -0.75 \\
$\left(\vartheta_{2}, x\right)$ & -0.93 & -0.86 & -0.75 & -0.75 \\
$\left(\vartheta_{2}, y\right)$ & -0.95 & -0.85 & -0.70 & -0.96 \\
$\left(\vartheta_{3}, x\right)$ & 0.05 & 0.25 & 0.60 & 0.65 \\
$\left(\vartheta_{3}, y\right)$ & -0.25 & 0.50 & 0.60 & -0.35 \\
$\left(\vartheta_{4}, x\right)$ & -0.35 & -0.05 & 0.30 & 0.30 \\
$\left(\vartheta_{4}, y\right)$ & -0.55 & 0.10 & 0.25 & 0.30 \\
$\left(\vartheta_{5}, x\right)$ & -0.50 & -0.25 & 0.10 & 0.15 \\
$\left(\vartheta_{5}, y\right)$ & -0.75 & -0.15 & 0.05 & -0.75 \\
$\left(\vartheta_{6}, x\right)$ & -0.98 & -0.94 & -0.93 & -0.94 \\
$\left(\vartheta_{6}, y\right)$ & -0.98 & -0.93 & -0.91 & -0.94 \\
$\left(\vartheta_{7}, x\right)$ & -0.93 & -0.91 & -0.86 & -0.91 \\
$\left(\vartheta_{7}, y\right)$ & -0.94 & -0.91 & -0.85 & -0.93 \\
$\left(\vartheta_{8}, x\right)$ & -0.93 & -0.91 & -0.84 & -0.86 \\
$\left(\vartheta_{8}, y\right)$ & -0.94 & -0.85 & -0.82 & -0.93 \\
$\left(\vartheta_{9}, x\right)$ & 0.80 & -0.35 & -0.65 & 0.05 \\
$\left(\vartheta_{9}, y\right)$ & 0.55 & -0.65 & -0.30 & -0.05 \\
$\left(\vartheta_{10}, x\right)$ & -0.85 & -0.60 & -0.25 & 0.05 \\
$\left(\vartheta_{10}, y\right)$ & -0.80 & -0.55 & -0.10 & 0.05 \\
\hline$\stackrel{\eta}{\eta}$ & -11.83 & -10.11 & -6.66 & -8.17 \\
\hline
\end{tabular}

TABLE 23: Final scores.

\begin{tabular}{|c|c|c|}
\hline$\dot{\xi}_{j}=\sum_{i} f_{i j}$ & $\dot{\eta}_{j}=\sum_{i} f_{i j}$ & $\dot{\circ}_{j}=\stackrel{\circ}{\xi}_{j}-\stackrel{\circ}{\eta}_{j}$ \\
\hline $\begin{array}{l}\xi_{1}=12.29 \\
\xi_{2}=10.21 \\
\xi_{3}=7.17 \\
\xi_{4}=9.30\end{array}$ & $\begin{array}{l}\stackrel{\circ}{1}_{1}=-11.83 \\
\stackrel{\eta}{\eta}_{2}=-10.11 \\
\grave{\eta}_{3}=-6.66 \\
\grave{\eta}_{4}=-8.17\end{array}$ & $\begin{array}{l}\stackrel{\stackrel{\lambda}{1}}{1}_{1}=24.12 \\
\stackrel{\lambda}{2}_{2}=20.32 \\
\grave{\lambda}_{3}=13.38 \\
\stackrel{\lambda}{\lambda}_{4}=17.47\end{array}$ \\
\hline
\end{tabular}

possibilities. Moreover, all these values remain the same for any sample. For instance, we considered a sample of 10,000 people in our assumptions.

The agencies analyze the tests and make data depending on the various kits and labs available in their respective areas and we get the following.

Tables 19 and 20 represent the information collected by the agencies regarding the tests in the form of agree FBSES and disagree FBSES, respectively.

Algorithm 1 based on FBSES is used to compare and rank the tests.

Using Tables 19 and 20, we calculate the focus agree and focus disagree or simply $f$-agree and $f$-disagree scores, which are displayed in Tables 21 and 22, respectively.

The final score table (see Table 23) indicates that the best available test is the rRT-PCR test. On the basis of the above calculations and assumptions, the tests are then ranked in their effectiveness as

rRT - PCR $>$ CBNAAT $>$ antibody test $>$ antigen test.

Remark 2. The above section gives a detailed comparison of the four widely used COVID-19 diagnosis tests, but the above details may vary with the original situation depending on the quality of kits, personnel, and availability of resources. Moreover, different techniques can prove to be much effective than others in different scenarios. For instance, RT-PCR takes more time; thus, for rapid testing, CBNAAT and antigen tests are more useful (though errorsome). For postdisease analysis, we use the antibody tests that detect the antibodies against COVID-19 created by the immune system of the body.

Figure 1 indicates how the sensitivity and specificity of tests vary with each other and with themselves in different conditions. In order to maximize the effectiveness, the quality of the tests must not be compromised as a slight 


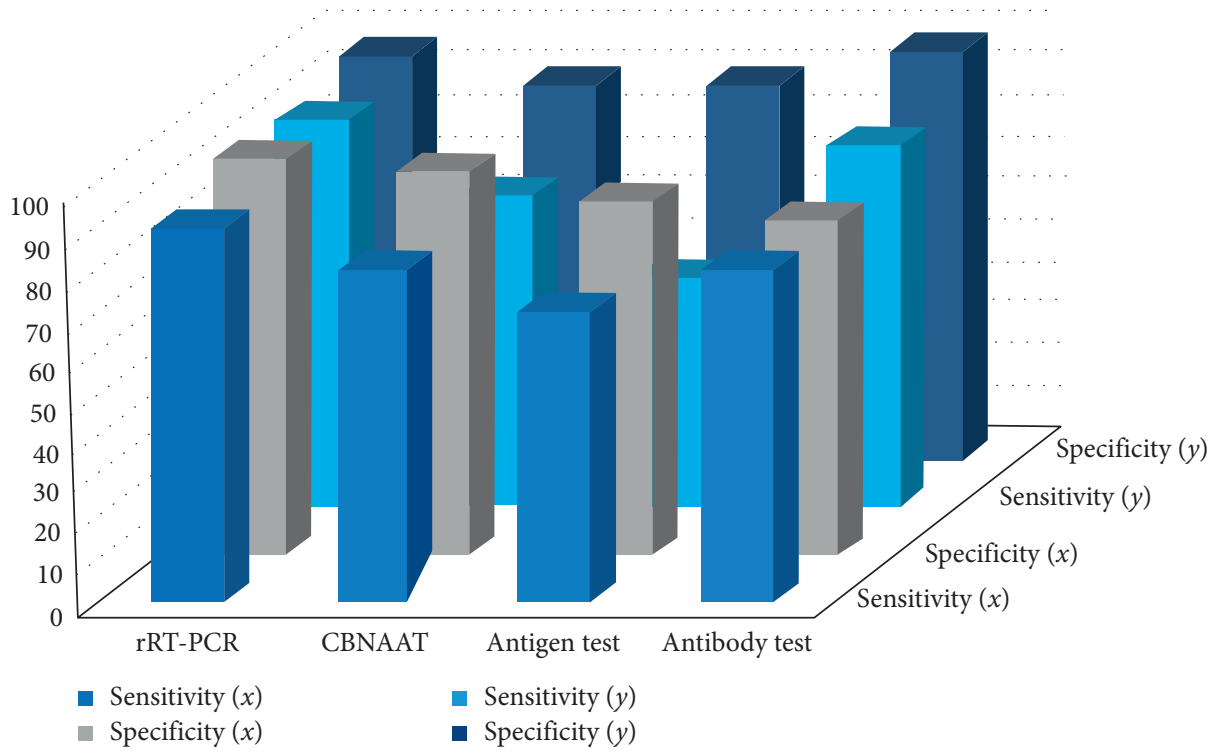

FIGURE 1: Sensitivity and specificity variations.

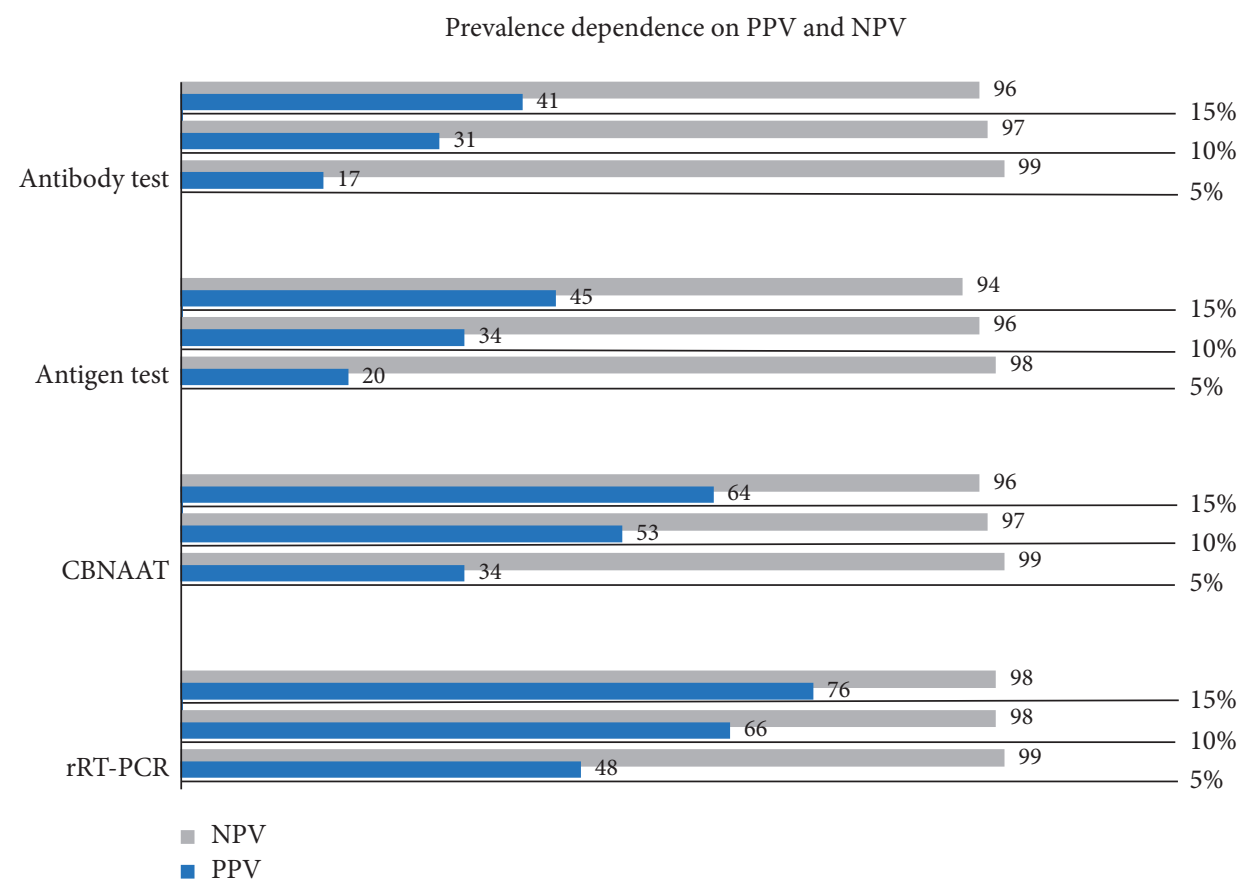

Figure 2: Prevalence dependence on PPV and NPV (at 5\%, 10\%, and 15\% prevalence of disease).

misinterpretation can prove to be disastrous on a large scale. Figure 2 shows the dependence of number of cases on the effectiveness of tests. Tests with the same specificity and sensitivity can show major differences with varying prevalences. With a slight increase or decrease in prevalence percentage, the number of false positives and false negatives can change significantly. Keeping this in view, prevalencedependent factors have been analyzed at three different levels. Despite the high test load, the rRT-PCR technique appeared to be the most effective in all situations and is therefore considered as the "gold standard" for testing.

In addition to the above diagnosis tests, contact tracing can also prove to be an effective strategy if implemented wisely. Countries such as China and South Korea used mobile tracing apps and geolocalization technology to announce high infection areas and track those in contact with the infected, thus getting a firm control on the spread of this disease efficiently. 
TABle 24: Comparison table for Example 10.

\begin{tabular}{lccc}
\hline Tests & Fuzzy SESs [15] & Fuzzy BSSs [9] & Proposed FBSESs \\
\hline$u_{1}$ & 12.26 & 43.00 & 24.12 \\
$u_{2}$ & 10.53 & 16.00 & 20.32 \\
$u_{3}$ & 7.22 & -32.00 & 13.83 \\
$u_{4}$ & 9.68 & -27.00 & 17.47 \\
\hline
\end{tabular}

TABLE 25: Comparison between ranking results of different models on Example 10.

\begin{tabular}{lc}
\hline Models & Ranking order \\
\hline Fuzzy SESs [15] & $u_{1}>u_{2}>u_{4}>u_{3}$ \\
Fuzzy BSSs [9] & $u_{1}>u_{2}>u_{4}>u_{3}$ \\
Proposed FBSESs & $u_{1}>u_{2}>u_{4}>u_{3}$ \\
\hline
\end{tabular}

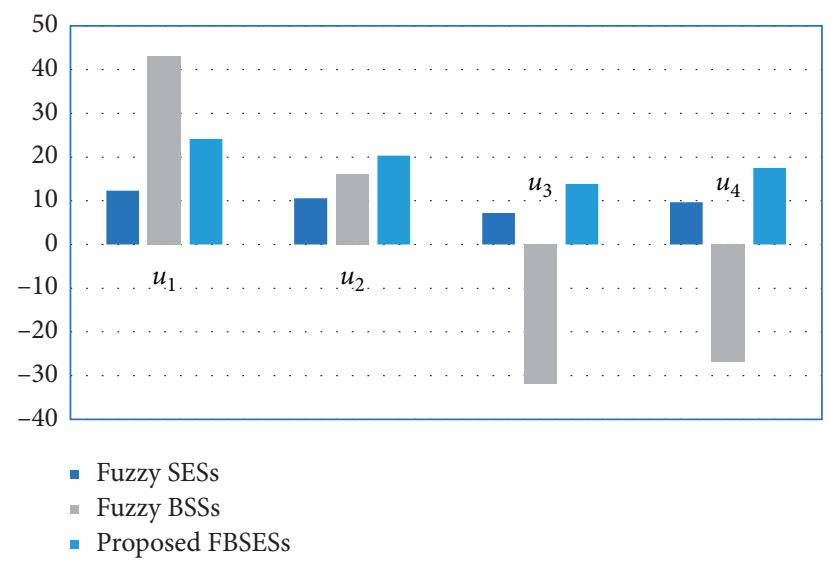

Figure 3: Comparison between results of different models on Example 10.

\section{Comparative Analysis}

To reveal the authenticity and viability of the developed FBSES model, in this section we discuss its advantages, limitations, and comparative analysis with fuzzy SESs and fuzzy BSSs.

(i) Advantages: last few decades have proved to be very productive in the decision sciences providing numerous powerful uncertain models to deal with uncertainties and vague information. More efficient uncertain models and their hybrid structures are to be proposed in order to deal with the uncertainties and novel problems arising in the world. The existing fuzzy BSSs [9] and fuzzy SESs [15] have proved their importance and effectiveness in dealing difficult uncertain situations, but a more viable model having mutual characteristics of both these models is required. For this reason in this study, we developed a novel hybrid model called FBSESs which is capable of dealing with information involving opinions of multiple experts in the fuzzy bipolar environment. Hence, the developed model is much authentic and viable in dealing MAGDM situations comparatively. It can be readily seen that the fuzzy BSSs cannot handle multiple expert opinions. Similarly, fuzzy SESs fail to handle parameters efficiently in a bipolar environment. Meanwhile, the proposed FBSES model is capable of handling both fuzzy bipolar soft information and fuzzy soft expert information collectively as well as individually.

(ii) Comparison: when dealing with MAGDM problems, models such as fuzzy SESs [15] and fuzzy BSSs [9] happen to be very productive in making correct decisions. However, they are restricted to be used in their respective environments because they can only deal with the information supported by their respective structures. This issue can be solved by combining two or more models, where the new hybrid model is formed by the combination of its parent models, which is more general and reliable than the former ones. The fuzzy SES model [15] is best suitable to deal with fuzzy soft expert information, but fails to handle fuzzy bipolar soft data. In a similar way, fuzzy BSSs [9] are capable of dealing with fuzzy bipolar soft information efficiently, but are inadequate in dealing with information concerning multiple expert opinions. This leads to the need of the FBSES model which can deal with fuzzy bipolar soft information under the opinion of multiple experts in one place. In order to proclaim the efficacy and reliability of the developed model, its comparative analysis with the fuzzy SESs [15] and fuzzy BSSs [9] is presented in Tables 24 and 25. For further clarification, Figure 3 is also provided to represent the comparison.

(iii) Limitations: the computational process of the developed model can be slow in the case of some MAGDM problems due to an increased number of parameters, as a result of the two oppositely defined sets of parameters viewed under the opinions of multiple experts. This limitation can be tackled if properly coded algorithm of the proposed model is practiced in different mathematical software such as MATLAB, which allows to deal with large datasets quickly and efficiently. Another important restriction is that the FBSES model is not very durable in the case of increased alternatives and/or parameters. Any increase or decrease in the alternatives can change the ranking order of the objects in a given MAGDM situation. The same is for the respective parameters. This is due to the independent behaviour of alternatives and parameters. 


\section{Conclusions}

Numerous real-world MAGDM problems from various domains, including medical sciences and artificial intelligence, have been solved efficiently by the fuzzy SES model and its generalizations. The fuzzy BSS model is another effective model inspiring many researchers in dealing situations with fuzzy bipolar soft information, and emerging as a key component in many hybrid models, including rough $m$-polar fuzzy BSSs, to tackle different uncertain situations. Despite their effectiveness, the abovementioned theories have their own limitations. The existing fuzzy SESs are not capable of handling situations concerning bipolar soft information, whereas the fuzzy BSS model is inadequate when considering multiple expert opinions. To overcome these limitations, this study presented the concept of FBSESs by combining fuzzy SESs and fuzzy BSSs which is more useful and reliable than its components. An important characteristic of this initiated model is its capability of dealing with fuzzy SES and fuzzy BSS information collectively as well as individually. In this research article, some fundamental properties of the proposed model, including subset, complement, extended union, extended intersection, restricted union, restricted intersection, AND operation, and OR operation have been discussed in detail. Moreover, a detailed comparison of the different types of COVID-19 tests and the ranking of their effectiveness in analyzing the spread of COVID-19 has been done under the novel FBSES model, which is supported by an efficient algorithm. Finally, we have provided a comparison of our initiated model with existing models such as fuzzy SESs [15] and fuzzy BSSs [9] to show the authenticity and supremacy of the developed hybrid model. In future, our research work can be expanded to (1) intuitionistic fuzzy BSESs, (2) $m$-polar fuzzy BSESs, (3) spherical fuzzy BSESs, (4) picture fuzzy BSESs, and (5) $q$-rung orthopair fuzzy BSESs.

\section{Data Availability}

No data were used to support this study.

\section{Ethical Approval}

This article does not contain any studies with human participants or animals performed by any of the authors.

\section{Conflicts of Interest}

The authors declare that they have no conflicts of interest.

\section{References}

[1] L. A. Zadeh, "Fuzzy sets," Information and Control, vol. 8, no. 3, pp. 338-353, 1965.

[2] W.-R. Zhang, "Bipolar fuzzy sets and relations: a computational framework for cognitive modeling and multiagent decision analysis," in Proceedings of the First International Joint Conference of The North American Fuzzy Information Processing Society Biannual Conference. The Industrial Fuzzy Control and Intellige, pp. 305-309, San Antonio, TX, USA, December 1994.
[3] K. T. Atanassov, "Intuitionistic fuzzy sets," Fuzzy Sets and Systems, vol. 20, no. 1, pp. 87-96, 1986.

[4] D. A. Molodtsov, "Soft set theory-first results," Computers and Mathematics with Applications, vol. 37, no. 4-5, pp. 19-31, 1999.

[5] P. K. Maji, A. R. Roy, and R. Biswas, "An application of soft sets in a decision-making problem," Computers and Mathematics with Applications, vol. 44, no. 8, pp. 1077-1083, 2002.

[6] M. I. Ali, F. Feng, X. Liu, W. K. Min, and M. Shabir, "On some new operations in soft set theory," Computers \& Mathematics with Applications, vol. 57, no. 9, pp. 1547-1553, 2009.

[7] P. K. Maji, R. Biswas, and A. R. Roy, "Fuzzy soft sets," Journal of Fuzzy Mathematics, vol. 9, no. 3, pp. 589-602, 2001.

[8] M. Shabir and M. Naz, "On bipolar soft sets," pp. 1303-1344, 2013, https://arxiv.org/abs/1303.1344.

[9] M. Naz and M. Shabir, "On fuzzy bipolar soft sets, their algebraic structures and applications," Journal of Intelligent \& Fuzzy Systems, vol. 26, no. 4, pp. 1645-1656, 2014.

[10] M. Akram and G. Ali, "Hybrid models for decision-making based on rough Pythagorean fuzzy bipolar soft information," Granular Computing, vol. 5, no. 1, pp. 1-15, 2020.

[11] M. Akram, G. Ali, and M. Shabir, "A hybrid decision-making framework using rough $\mathrm{mF}$ bipolar soft environment," Granular Computing, vol. 6, no. 2021, pp. 539-555.

[12] X. Ma, Q. Liu, and J. Zhan, "A survey of decision making methods based on certain hybrid soft set models," Artificial Intelligence Review, vol. 47, no. 4, pp. 507-530, 2017.

[13] T. Mahmood, "A novel approach towards bipolar soft sets and their applications," Journal of Mathematics, vol. 2020, Article ID 4690808, 11 pages, 2020.

[14] S. Alkhazaleh and A. R. Salleh, "Soft expert sets," Advances in Decision Sciences, vol. 2011, Article ID 757868, 12 pages, 2011.

[15] S. Alkhazaleh and A. R. Salleh, "Fuzzy soft expert set and its application," Applied Mathematics, vol. 5, no. 9, pp. 1349-1368, 2014.

[16] F. Adam and N. Hassan, "Multi Q-fuzzy soft expert set and its application," Journal of Intelligent \& Fuzzy Systems, vol. 30, no. 2, pp. 943-950, 2016.

[17] Y. A. Qudah and N. Hassan, "Bipolar fuzzy soft expert set and its application in decision making," International Journal of Applied Decision Sciences, vol. 10, no. 2, pp. 175-191, 2017.

[18] I. Arockiarani and A. Arokia Lancy, "Multi criteria decision making problem with soft expert set," International Journal of Computer Applications, vol. 78, no. 15, pp. 1-4, 2013.

[19] M. Bashir and A. R. Salleh, "Fuzzy parameterized soft expert set," Abstract and Applied Analysis, vol. 2012, Article ID 258361, 15 pages, 2012.

[20] M. Bashir and A. R. Salleh, "Possibility fuzzy soft expert set," Open Journal of Applied Sciences, vol. 2, no. 4, pp. 208-211, 2012.

[21] A. Qayyum, S. Abdullah, and M. Aslam, "Cubic soft expert sets and their application in decision making," Journal of Intelligent \& Fuzzy Systems, vol. 31, no. 3, pp. 1585-1596, 2016.

[22] S. Broumi and F. Smarandache, "Intuitionistic fuzzy soft expert sets and its application in decision making," Journal of New Theory, vol. 1, pp. 89-105, 2015.

[23] N. Hassan and K. Alhazaymeh, "Vague soft expert set theory," AIP Conference Proceedings, vol. 1522, no. 1, pp. 953-958, 2013.

[24] M. Shabir and R. Gul, "Modified rough bipolar soft sets," Journal of Intelligent \& Fuzzy Systems, vol. 39, no. 3, pp. 4259-4283, 2020.

[25] G. Ali and M. Akram, "Decision-making method based on fuzzy N-soft expert sets," Arabian Journal for Science and Engineering, vol. 45, no. 12, pp. 10381-10400, 2020. 
[26] M. Akram, G. Ali, M. A. Butt, and J. C. R. Alcantud, "Novel MCGDM analysis under $m$ - polar Fuzzy soft expert sets," Neural Computing and Applications, 2021.

[27] A. Adeel, M. Akram, N. Yaqoob, and W. Chammam, "Detection and severity of tumor cells by graded decision-making methods under fuzzy $N$-soft model," Journal of Intelligent \& Fuzzy Systems, vol. 39, no. 1, pp. 1303-1318, 2020.

[28] M. Akram, A. Adeel, A. N. Al-Kenani, and J. C. R. Alcantud, "Hesitant fuzzy N-soft ELECTRE-II model: a new framework for decision-making," Neural Computing and Applications, vol. 33, pp. 1-16, 2020.

[29] M. Akram, A. Adeel, and J. C. R. Alcantud, "Group decisionmaking methods based on hesitant $N$-soft sets," Expert Systems with Applications, vol. 115, pp. 95-105, 2019.

[30] M. Akram, F. Ilyas, and H. Garg, "Multi-criteria group decision making based on ELECTRE I method in Pythagorean fuzzy information," Soft Computing, vol. 24, no. 5, pp. 3425-3453, 2020.

[31] M. Irfan Ali, "A note on soft sets, rough soft sets and fuzzy soft sets," Applied Soft Computing, vol. 11, no. 4, pp. 3329-3332, 2011.

[32] G. Ali, M. Akram, and J. C. R. Alcantud, "Attributes reductions of bipolar fuzzy relation decision systems," Neural Computing and Applications, vol. 32, no. 14, pp. 10051-10071, 2020.

[33] C. Jana, G. Muhiuddin, and M. Pal, "Multi-criteria decision making approach based on SVTrN dombi aggregation functions," Artificial Intelligence Review, vol. 54, no. 2021, pp. 3685-3723.

[34] C. Jana, G. Muhiuddin, and M. Pal, "Multiple-attribute decision making problems based on SVTNH methods," Journal of Ambient Intelligence and Humanized Computing, vol. 11, no. 9, pp. 3717-3733, 2020.

[35] F. Feng, C. Li, B. Davvaz, and M. I. Ali, "Soft sets combined with fuzzy sets and rough sets: a tentative approach," Soft Computing, vol. 14, no. 9, pp. 899-911, 2010.

[36] F. Feng and Y. Li, "Soft subsets and soft product operations," Information Sciences, vol. 232, pp. 44-57, 2013.

[37] F. Karaaslan and S. Karataş, "A new approach to bipolar soft sets and its applications," Discrete Mathematics, Algorithms and Applications, vol. 7, no. 4, p. 1550054, 2015.

[38] N. Malik and M. Shabir, "Rough fuzzy bipolar soft sets and application in decision-making problems," Soft Computing, vol. 23, no. 5, pp. 1603-1614, 2019.

[39] G. Shahzadi, G. Muhiuddin, M. Arif Butt, and A. Ashraf, "Hamacher interactive hybrid weighted averaging operators under Fermatean fuzzy numbers," Journal of Mathematics, vol. 2021, Article ID 5556017, 17 pages, 2021. 